\title{
Choice activity stabilizes sensory representations and mediates sensorimotor associations in parietal cortex
}

Ting-Yu Chang ${ }^{1 \dagger}$, Raymond Doudlah ${ }^{1 \dagger}$, Byounghoon Kim ${ }^{1}$, Adhira Sunkara ${ }^{2}$, Meghan Lowe ${ }^{1}$, and Ari Rosenberg ${ }^{1}$

${ }^{1}$ Department of Neuroscience

'Department of Surgery

School of Medicine and Public Health

University of Wisconsin - Madison,

Madison, WI. 53705, USA.

tEqual Contributions

\section{Correspondence}

Ari Rosenberg

Department of Neuroscience

School of Medicine and Public Health

University of Wisconsin - Madison

1111 Highland Ave. WIMR-II, Office 5505

Madison, WI. 53705

Email: ari.rosenberg@wisc.edu

\section{Keywords}

Sensorimotor, choice activity, parietal cortex, vision, stereopsis, perspective, 3D pose, slant, tilt, orientation, distance, depth, oculomotor, saccade, marginalization, nuisance variables 


\section{Abstract}

Selecting actions which achieve desired goals often requires three-dimensional (3D) representations of the environment. Because the sensory epithelia cannot directly encode the world's 3D spatial features, sensory signals must be converted into 3D representations. Here we investigated the relationships between the quality of 3D visual representations, choice-related activity, and motor-related activity in the parietal cortex of macaque monkeys using an eightalternative 3D orientation discrimination task, visually guided saccade task, and laminar probe recordings. We found that choice activity was preferentially carried by caudal intraparietal area neurons with more robust 3D representations. Choice activity further stabilized the 3D representations, rather than attenuating information not directly relevant to the behavioral task (nuisance variables). An experience-dependent, sensorimotor association additionally aligned sensory and saccade direction preferences, particularly for neurons with choice activity. These findings reveal novel roles for choice activity in improving the fidelity of ecologically relevant object representations and mediating sensorimotor associations.

\section{Introduction}

Interactions with the environment require that sensory information be mapped to motor responses.

The parietal cortex is an important site of sensorimotor transformations (Rushworth et al., 1997;

in deficits associated with the impaired use of sensory information to create and execute motor plans, as opposed to deficits in sensory processing or action (Pause and Freund, 1989; Pause et al., 1989). They can also produce 3D visual processing deficits (Holmes, 1918; Holmes and Horrax, 1919). Although sensorimotor transformations are often studied using two-dimensional (2D) stimulus paradigms, mappings between 3D object information and motor responses are essential in the natural world. Thus, parietal cortex may have a fundamental role in creating robust $3 \mathrm{D}$ representations and mapping them to specific actions.

Within parietal cortex, an important site of $3 D$ sensory processing is the caudal intraparietal (CIP) area. Neurons in CIP are tuned for 3D orientation (Taira et al., 2000; Rosenberg et al., 2013) signaled by multiple cues (Tsutsui et al., 2001; Tsutsui et al., 2002; Rosenberg and

62 Angelaki, 2014b), and perform multisensory processing to achieve gravity-centered object 63 representations (Rosenberg and Angelaki, 2014a). In addition to carrying high-level sensory 64 representations, CIP activity correlates with the short-term memory and perceptual matching of 65 3D features (Tsutsui et al., 2003), and choices made during a binary orientation discrimination 66 task (Elmore et al., 2019). Furthermore, inactivating CIP impairs 3D feature discrimination 
(Tsutsui et al., 2001; Van Dromme et al., 2016).

The anatomical projections and effective connectivity of CIP suggest that it may also contribute to goal-directed sensorimotor transformations (Nakamura et al., 2001; Premereur et al., 2015; Van Dromme et al., 2016; Lanzilotto et al., 2019). In particular, CIP projects to areas involved in motor planning and execution, including the lateral intraparietal area (LIP) (Andersen et al., 1992; Bennur and Gold, 2011; Shushruth et al., 2018), anterior intraparietal area (Murata et al., 2000; Baumann et al., 2009; Pani et al., 2014), and V6A (Fattori et al., 2010; Fattori et al., 2012; Breveglieri et al., 2016). However, it is untested if CIP carries motor-related signals, or if its output only contains 3D sensory information.

Here we investigated the relationships between sensory representations, choice-related activity, and motor-related activity in CIP. Neuronal activity was recorded while macaque monkeys performed an eight-alternative forced choice (8AFC) tilt discrimination task with planar surfaces presented at different slants and distances (Chang et al., 2020). The neurons differed in the extent to which their 3D orientation selectivity depended on distance. Choice activity was preferentially carried by neurons with more robust 3D representations, and further stabilized 3D selectivity. Choice tuning was parametric, and the choice and tilt preferences aligned. Motor-related activity was assessed using a visually guided saccade task (Munoz and Wurtz, 1995; Hanes and Schall, 1996). We found that many CIP neurons had saccade direction tuning. Moreover, the surface tilt and saccade direction preferences were mapped onto one another, reflecting a sensorimotor association that was strongest for neurons with choice activity.

These results identify systematic relationships between sensory representations, choicerelated activity, and motor-related activity. Neurons that robustly represented the task-relevant information were most strongly coupled with the choices. While choice activity is widely associated with decision processes, the current results reveal novel roles in stabilizing sensory representations and mediating sensorimotor associations. The coupling of sensory and motor functions by single neurons may facilitate sensorimotor transformations over short timescales that promote successful interactions with the environment.

\section{Results}

We tested three hypotheses about the relationships between sensory representations, choicerelated activity, and motor-related activity. First, neurons with more robust 3D representations would preferentially carry choice activity. Second, choice activity would stabilize 3D selectivity, as opposed to attenuating information that was not directly relevant to the task at hand (i.e., nuisance variables). Third, sensorimotor associations would be mediated by choice activity. 
101

102

103

104

105

106

107

\section{Quantifying 3D tilt sensitivity}

Testing our hypotheses required a 3D visual discrimination task that could also be used to quantify the robustness of neuronal 3D representations. To this end, we trained two monkeys to perform an 8AFC orientation discrimination task under different viewing conditions that determined the task difficulty (Chang et al., 2020). The task required the monkeys to report which side of a plane was nearest (i.e., the plane's tilt) through a saccade to one of eight choice targets (Figure 1A).

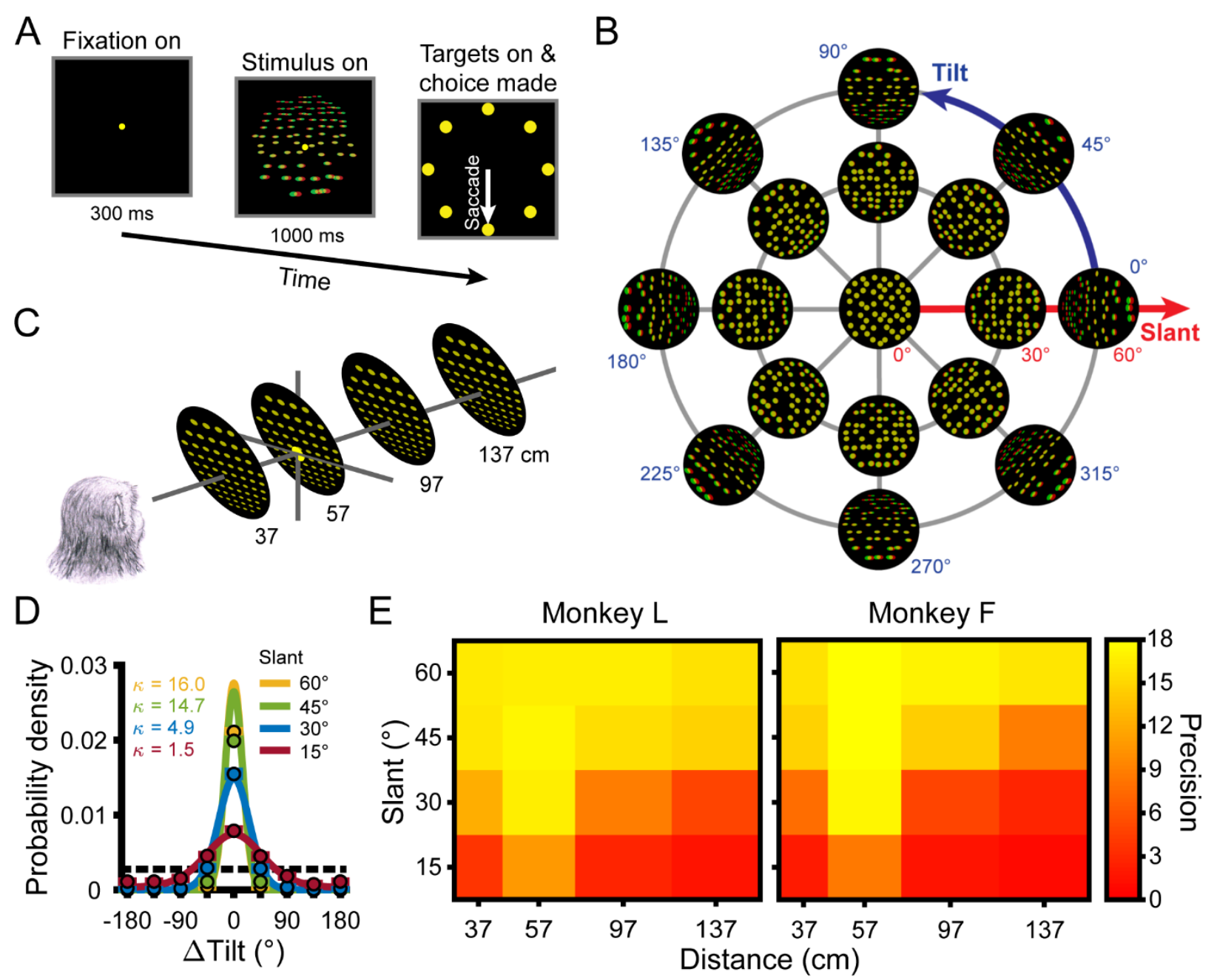

Figure 1. Task, stimuli, and performance. (A) Tilt discrimination task. After fixating a target at the center of the screen for $300 \mathrm{~ms}$, a plane was shown for $1 \mathrm{~s}$ while fixation was maintained. The fixation target and plane then disappeared and eight choice targets appeared. The plane's tilt was reported via a saccade to the corresponding target (e.g., the bottom target for a bottom-near plane). (B) Tilt and slant. Planes were rendered as random dot stereograms with perspective and stereoscopic cues. A subset of planes are shown here as red-green anaglyphs. For clarity, the number of dots is reduced and the dot size is increased compared to the actual stimuli. (C) The planes subtended $20^{\circ}$ of visual angle, and were presented at the center of the screen at four distances. The fixation point (depicted here as a larger yellow dot) was always located at $57 \mathrm{~cm}$. (D) Error distributions of reported tilts for Monkey $L$ at each slant (distance $=137 \mathrm{~cm}$ ), calculated over all tilts. Data points show the mean probability of a given $\Delta$ Tilt (correct choice: $\Delta$ Tilt $=0^{\circ}$ ) and 
SEM (error bars are obscured by the data points) across sessions. Solid curves are von Mises probability density function fits. Taller and narrower densities indicate greater sensitivity. Sensitivities ( $\kappa$, the von Mises concentration parameter) are indicated in the inset. At higher sensitivities, deviation between the data point at $\Delta \mathrm{Tilt}=0^{\circ}$ and the density function reflect discrete versus continuous representations of the area between sampled tilts (Chang et al., 2020). Chance performance is marked by the dashed horizontal line. (E) Heat maps show mean tilt sensitivity across sessions as a function of slant and distance for each monkey. Yellow hues indicate greater sensitivity.

Tilt and slant are polar coordinates describing 3D surface orientation (Figure 1B). Tilt is the angular variable $\left(0^{\circ} \leq \mathrm{T}<360^{\circ}\right)$ and specifies the direction that the plane is oriented in depth. For example, $\mathrm{T}=0^{\circ}$ indicates right-near and $\mathrm{T}=90^{\circ}$ indicates top-near. Planes were presented at 8 tilts $\left(0^{\circ}\right.$ to $315^{\circ}, 45^{\circ}$ steps $)$, corresponding to the 8 choice options. Slant is the radial variable $\left(0^{\circ} \leq \mathrm{S} \leq 90^{\circ}\right)$ and specifies the amount of depth variation. There is no depth variation at $\mathrm{S}=0^{\circ}$, so tilt is undefined and there is no correct task response. Larger slants indicate greater depth variation. Planes were presented at 5 slants $\left(0^{\circ}\right.$ to $60^{\circ}, 15^{\circ}$ steps $)$. Each of the 33 orientations was presented at 4 distances: 37, 57, 97, and $137 \mathrm{~cm}$ (Figure 1C). Thus, 132 unique surface poses were shown.

Behavioral performance was quantified at each combination of tilt, slant, and distance using the distribution of reported tilt errors ( $\Delta$ Tilt $=$ reported tilt - presented tilt) each session (Monkey L: $\mathrm{N}=26$ sessions; Monkey F: $\mathrm{N}=27$ ) (Chang et al., 2020). Sensitivity was defined as the concentration parameter $(\kappa)$ of the von Mises probability density function fit to the distribution of reported tilt errors (equation 1). For both monkeys, we found that sensitivity significantly depended on slant (generalized linear regression; both $p$-values $\leq 2.9 \times 10^{-28}$ ) and distance (both $p$-values $\leq 2.7 \times 10^{-5}$ ), but not tilt (linearized into cosine and sine components; all four $p$-values $\geq$ 0.19) (Fisher, 1995). We therefore summarized sensitivity as a function of slant and distance calculated over all tilts (Figure 1D, Figure 1-figure supplement 1). Consistent with our previous findings in which behavioral performance in the 8AFC tilt discrimination task was extensively analyzed over a wide range of 3D poses as well as multiple visual cue conditions (Chang et al., 2020), we found that sensitivity decreased with distance from fixation $(57 \mathrm{~cm})$ and increased with slant (Figure 1E). 


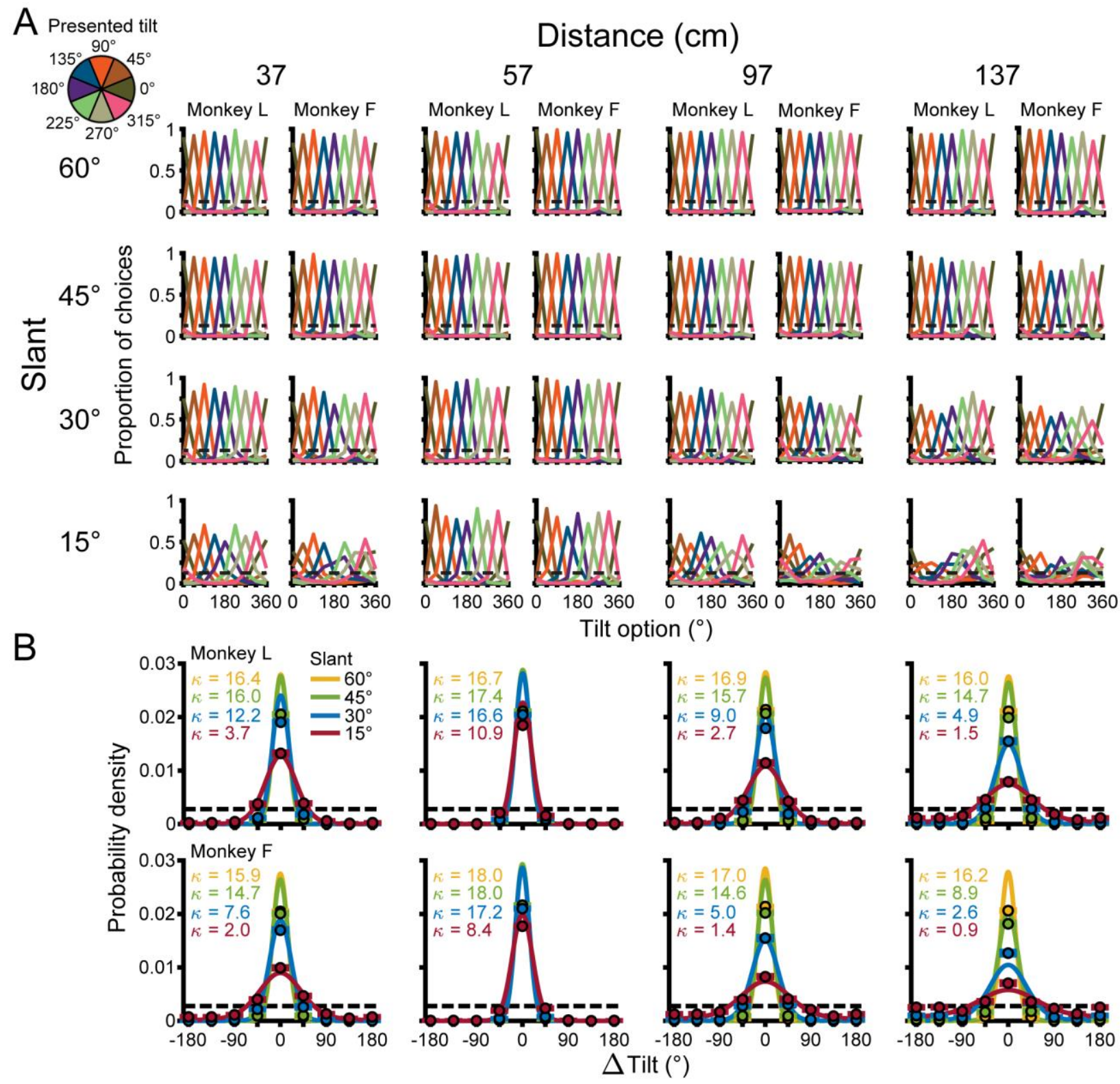

Figure 1-figure supplement 1. Behavioral performance. (A) Proportion of choices made for each tilt option at each combination of tilt (colors), slant (rows), and distance (supercolumns), and each monkey (subcolumns). Chance performance is marked by dashed horizontal lines. Performance was near perfect at combinations of large slants and distances near fixation $(57 \mathrm{~cm})$, but approached chance level at small slants and distances far from fixation. Sensitivity was not significantly dependent on tilt (see text for details). (B) Error distributions of reported tilts at each combination of slant (colors) and distance (columns), calculated over all tilts (Monkey L: top row; Monkey F: bottom row). Data points show the mean probability of a given $\Delta$ Tilt (correct choice: $\Delta$ Tilt $=0^{\circ}$ ) and SEM (error bars are obscured by the data points) across sessions. Solid curves are von Mises probability densities. Sensitivities are indicated in the insets. Chance performance is marked by dashed horizontal lines. 


\section{CIP responses to 3D surface pose predict tilt sensitivity}

We next assessed if the sensory representations in CIP accounted for the monkeys' tilt sensitivity. While the monkeys performed the task, we recorded from 437 neurons (Monkey L: $N=218$; Monkey $\mathrm{F}: \mathrm{N}=219$ ) using laminar probes (Figure 2). Because CIP neurons can carry sensory and choice-related signals (Elmore et al., 2019), we divided the responses into two time windows. The "sensory only" (SO) window started at the median visual response latency (52 ms) and ended at the onset of choice activity (202 ms; calculated below). The "sensory plus choice" (SPC) window, which can include both sensory and choice activity, started at the onset of choice activity and ended at the stimulus offset (1 s). We first analyze responses in the SO window.
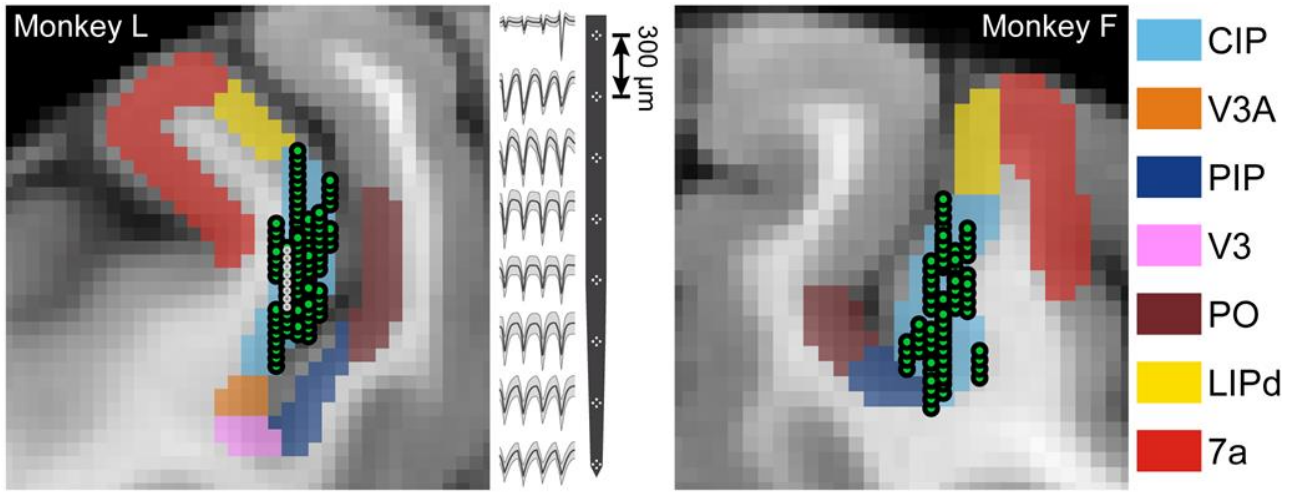

Figure 2. Neuronal recordings. Coronal MRI sections showing the estimated boundaries of CIP (light blue) and neighboring regions (see Materials and methods). All recording locations (green circles) are shown, projected along the anterior-posterior (AP) axis onto a single section (Monkey $\mathrm{L}: \mathrm{AP}=-7 \mathrm{~mm}$; Monkey $\mathrm{F}: \mathrm{AP}=-5.5 \mathrm{~mm}$ ). Spike waveforms from an eight-tetrode recording (locations marked by smaller white circles in the MRI) are shown for Monkey L.

Distinguishing 3D representations from sensitivity to lower-level features (e.g., local binocular disparity cues) that co-vary with changes in object pose is a longstanding problem (Janssen et al., 2000; Nguyenkim and DeAngelis, 2003; Alizadeh et al., 2018; Elmore et al., 2019). These possibilities can be disentangled by assessing how orientation selectivity is affected by the object's distance when the fixation distance is held constant. A 3D representation is indicated if the orientation selectivity (tuning curve shape) is invariant to the distance (though the gain can change). In contrast, sensitivity to lower-level features is implied if the selectivity changes drastically. The sensory responses of five CIP neurons that portray the range of sensory, choicerelated, and motor-related properties we found are shown in Figure 3.

In an idealized 3D representation, the orientation tuning (preference and bandwidth, but not gain) is independent of distance. That is, 3D pose tuning will be separable over orientation and distance. To quantify how well CIP activity conformed to this ideal, we fit each neuron's 
responses with a separable model (equation 7). A tolerance index was then defined as the average correlation between the responses and fit at each distance. The index quantifies, on a continuum, if a neuron is more selective for 3D pose (values closer to 1) or lower-level visual features (values closer to 0 ). Some neurons were highly tolerant, indicating robust 3D tuning (Figure 3A; Tolerance $=0.88$ ). Other neurons had modest tolerances, suggesting intermediate representations between 3D tuning and lower-level feature selectivity (Figure 3B; Tolerance = 0.53). Still others had low tolerances, consistent with lower-level feature selectivity (Figure 3C,D; Tolerance $=0.34$ and 0.24 , respectively). A minority had stronger distance selectivity than orientation selectivity (Figure 3E). Across the population, 62 neurons (14\%) were distance selective (two-way ANOVA, $p<0.05)$ but not orientation selective $(p \geq 0.05)$.
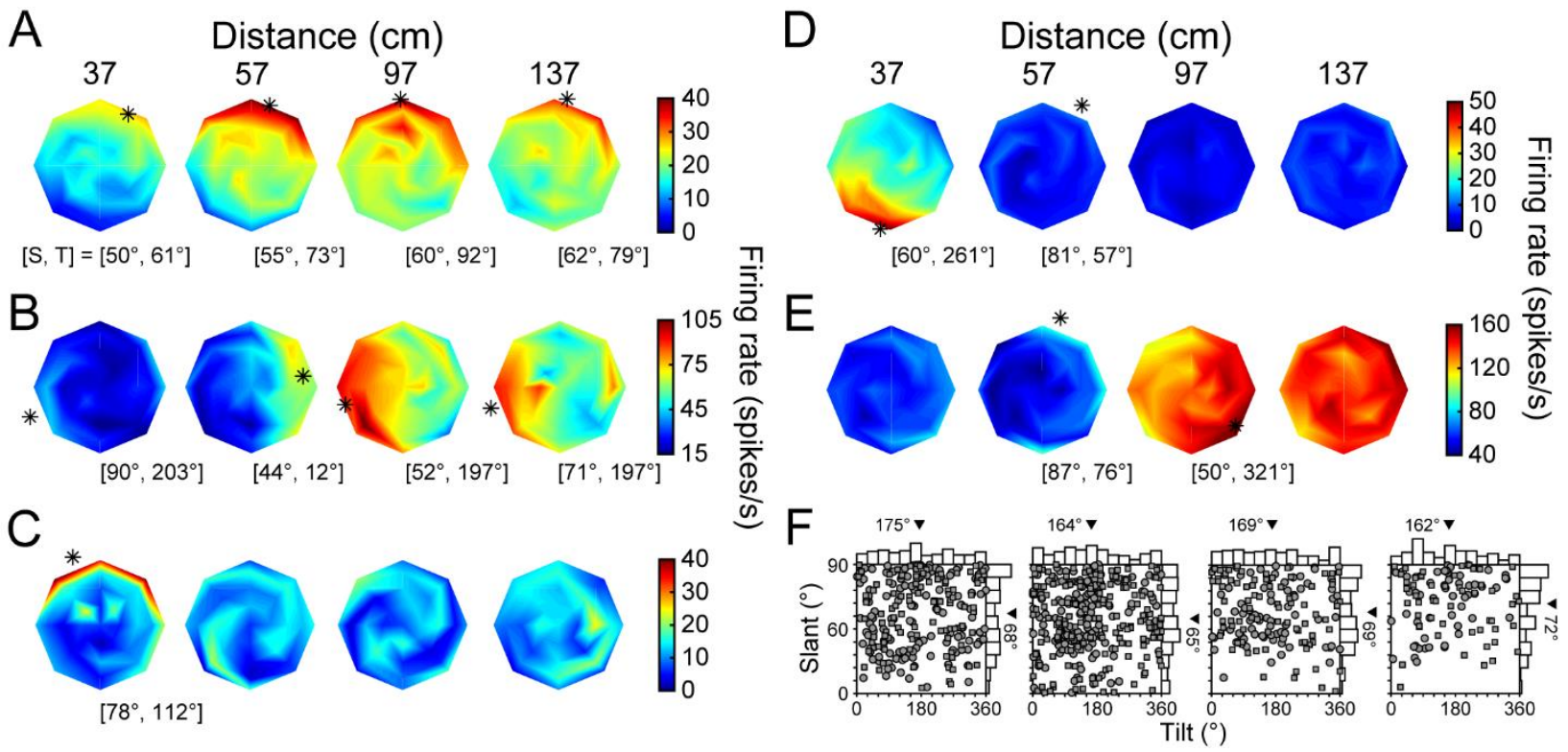

Figure 3. 3D orientation tuning at each distance before the onset of choice activity. (A-E) Five example neurons. Heat maps show firing rate as a function of tilt (T) and slant (S), plotted using the coordinates illustrated in Figure 1B. Red hues indicate higher firing rates. Asterisks mark preferred orientations from Bingham fits (tuned cases only). Some asterisks are not on the discs because the largest tested slant was $60^{\circ}$. (A) High tolerance neuron. (B) Intermediate tolerance neuron. (C) Low tolerance neuron. (D) Low tolerance neuron. (E) Distance selective neuron with little orientation-related modulation. (F) Distribution of tilt and slant preferences at each distance plotted using an equal area projection (Monkey L: circles; Monkey F: squares). Only tuned neurons at each distance are included: $37(\mathrm{~N}=242), 57(\mathrm{~N}=320), 97(\mathrm{~N}=158), 137(\mathrm{~N}=106)$ $\mathrm{cm}$. Marginal histograms show the tilt and slant distributions. Triangles mark mean values.

Vergence eye movements are a potential factor that can influence neuronal responses. To evaluate if our measurements of 3D pose tuning were affected by vergence, we performed an analysis of covariance on the responses of each neuron with tilt (linearized into cosine and sine components), slant, and distance as independent factors and the mean vergence over the 
analyzed time window as a covariate (DeAngelis and Uka, 2003). Both the SO and SPC windows were included in this analysis because the results were similar for the individual time windows. For only 40 neurons (9\%) was there a statistically significant effect of vergence $(p<0.05)$. Moreover, the significance of the tilt, slant, and distance main effects was unchanged for all but 11 neurons (3\%) when vergence was included as a covariate. These effects are smaller than those previously reported for disparity tuning in the middle temporal area (DeAngelis and Uka, 2003), and indicate that vergence had a minimal impact on the 3D pose tuning.

We next evaluated if the distribution of orientation preferences during the SO window was distance dependent. Preferences were estimated by fitting a Bingham function to each significant orientation tuning curve (ANOVA, $p<0.05$, with Bonferroni-Holm correction for each neuron) (Rosenberg et al., 2013). The distribution of tilt preferences was not significantly different from uniform at any distance $\left(\chi^{2} ; 37,57\right.$, and $97 \mathrm{~cm}$ : $p$-values $\geq 0.43 ; 137 \mathrm{~cm}: p=0.03$, not significant after Bonferroni-Holm correction). At $57 \mathrm{~cm}$ (fixation distance), the distribution of slant preferences was not significantly different from uniform $(p=0.13)$. These findings are consistent with our previous results. However, the distributions of slant preferences were significantly different from uniform at 37, 97, and $137 \mathrm{~cm}$ (all $p$-values $\leq 1.2 \times 10^{-3}$, with Bonferroni-Holm correction). At each of these distances, the mean slant preference was larger than at $57 \mathrm{~cm}$ (Figure 3F).

To determine why the slant preferences were larger at distances closer and further than fixation, we performed pairwise comparisons of the slant preferences of neurons with orientation tuning at adjacent distances. Preferences at 37 and $57 \mathrm{~cm}$ were not significantly different (paired t-test, $p=0.19, \mathrm{~N}=209$ ). For that subset of neurons, we therefore examined the distribution of preferences at $57 \mathrm{~cm}$ and found that it was significantly different from uniform $\left(\chi^{2}, p=1.5 \times 10^{-2}\right)$. In contrast, the distribution of preferences for the 111 neurons that represented 57 but not $37 \mathrm{~cm}$ was not different from uniform $(p=0.24)$. Thus, neurons that represented $37 \mathrm{~cm}$ tended to prefer larger slants. We further found that the preferences of individual neurons tended to increase from 57 to $97 \mathrm{~cm}$ (mean $\Delta$ Slant $=5.1^{\circ}$; paired t-test, $p=1.1 \times 10^{-3}, \mathrm{~N}=144$ ), as well as from 97 to 137 $\mathrm{cm}$ (mean $\Delta$ Slant $=4.3^{\circ} ; p=9.7 \times 10^{-3}, \mathrm{~N}=87$ ). Thus, there was a slight inseparability in the sensory tuning such that the slant preferences tended to increase with distance behind fixation. These results are consistent with the behavioral data which showed that sensitivity fell off more slowly with distance from fixation at larger compared to smaller slants (Figure 1E).

We previously found that behavioral tilt sensitivity could be explained by a neuronal probabilistic population code model of perspective and stereoscopic cue integration (Chang et al., 2020). The model predicted a monotonic relationship between behavioral sensitivity and neuronal response gain. We tested this prediction by comparing the tilt sensitivity at each slant-distance 
combination to the corresponding gain of the tilt tuning curves averaged over all neurons (Figure 4). Consistent with the prediction, tilt sensitivity increased as a function of response gain (Monkey $\mathrm{L}$ : Spearman $r=0.95, p=2.2 \times 10^{-308}$; Monkey $\mathrm{F}: r=0.87, p=2.2 \times 10^{-308}$ ). The finding that response gain was highly predictive of behavioral sensitivity across performance levels ranging from nearchance to near-perfect (Figure 1E, Figure 1-figure supplement 1) suggests that CIP activity may constrain the precision of tilt perception.

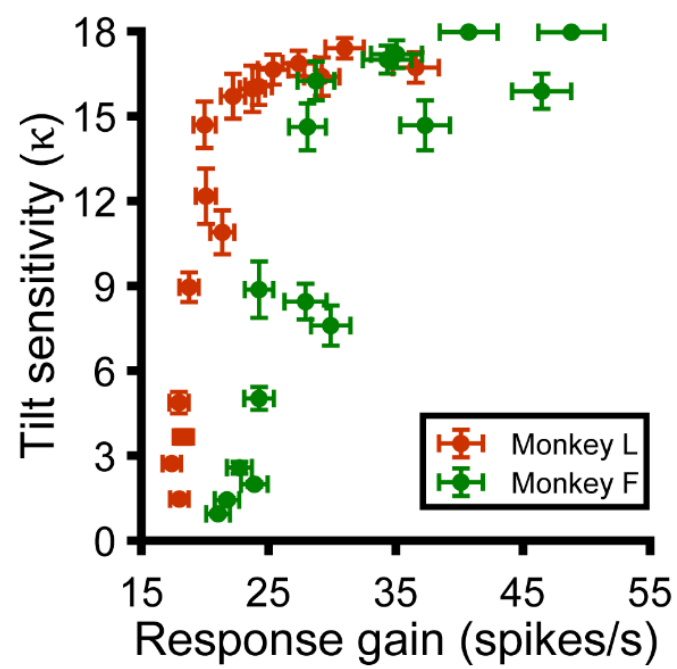

Figure 4. Neuronal response gain predicts behavioral tilt sensitivity. Tilt sensitivity $(\kappa)$ versus response gain for each slant-distance combination (Monkey L: orange; Monkey F: green). Data points show mean and SEM across sessions or neurons. Sensitivity does not exceed $\kappa=18$, the upper limit that can be estimated with a $45^{\circ}$ tilt sampling interval (Chang et al., 2020).

\section{Choice activity was parametrically tuned and aligned with the sensory preferences}

We next tested each neuron for choice-related activity. An advantage of the 8AFC task was that it could reveal parametric choice tuning, which is not possible with a typical binary choice task. To test for choice-related activity, we only used frontoparallel plane $\left(S=0^{\circ}\right)$ responses since tilt is undefined at that orientation. To increase the statistical power, we combined responses across all distances after separately z-scoring them at each distance. Responses were then grouped according to the choice that was made on each trial.

We computed eight population-level time courses of choice-related activity, relative to the choice that elicited the maximum response for each neuron (Figure 5A). The time course of choice activity had parametric tuning, with an amplitude that symmetrically decreased with greater deviation from the preferred choice (note the similarity of the $\pm 45^{\circ}, \pm 90^{\circ}$, and $\pm 135^{\circ}$ curves). The onset of choice activity was defined as the first time point after stimulus onset that the curves significantly diverged (ANOVA, $p<0.05$ ). All choice analyses were performed using the SPC 

SO window. Across the population, 201 neurons (46\%) had choice activity (ANOVA, $p<0.05$ ).

283
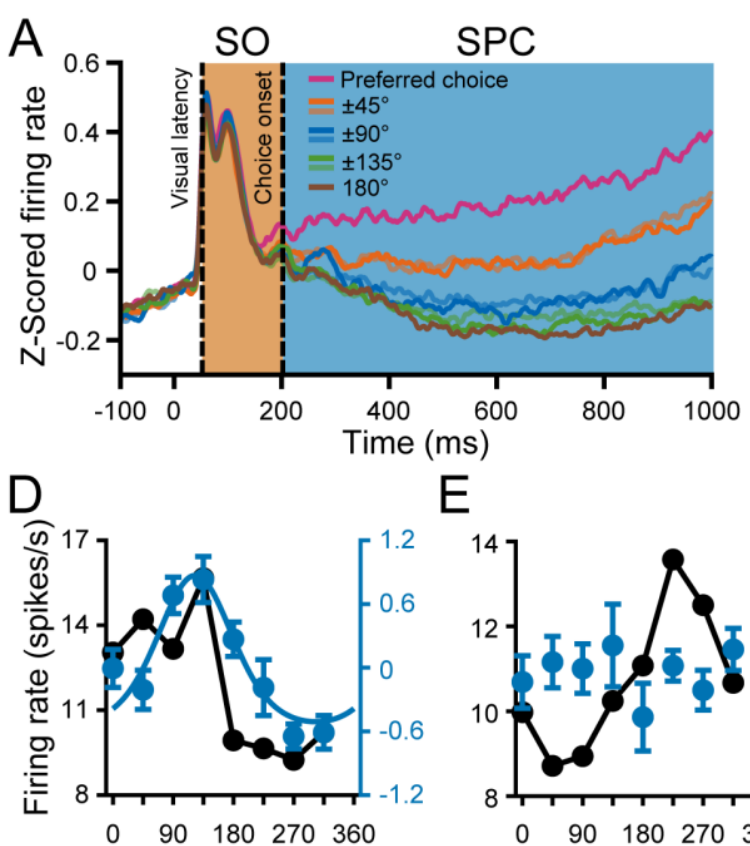

E

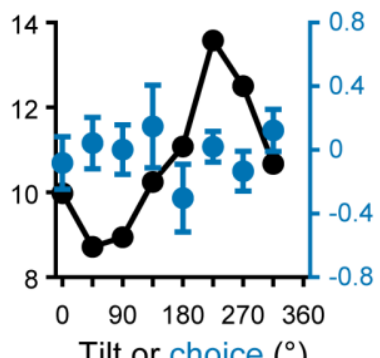

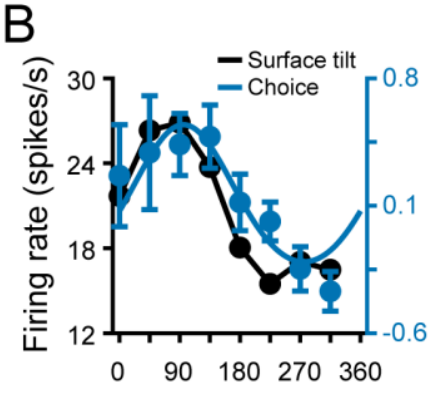

Tilt or choice $\left({ }^{\circ}\right)$

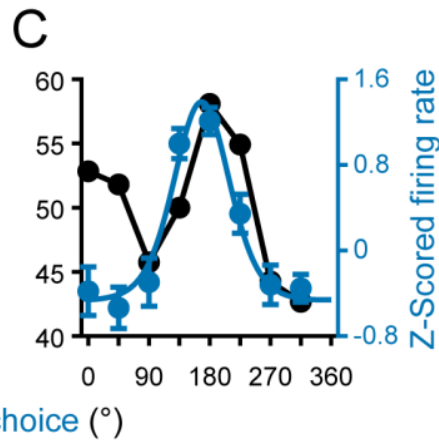

$\mathrm{F}$

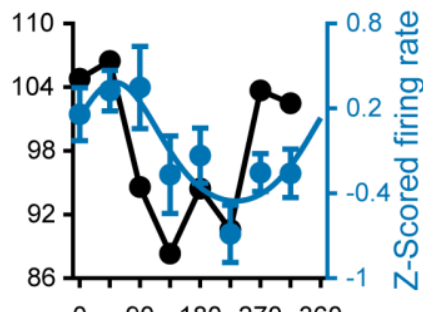

$\begin{array}{lllll}0 & 90 & 180 & 270 & 360\end{array}$

G

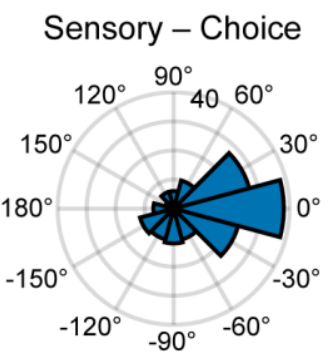

284

285

286

287

288

289

290

291

292

293

294

295

296

297

298

299

300

301

302

303

Figure 5. Choice tuning was parametric and aligned with the sensory preferences. (A) Time course of choice-related activity. Curves are average z-scored responses for each of the eight choices relative to the preferred choice. Stimulus onset $=0 \mathrm{~ms}$. Vertical dashed lines mark the response latency $(52 \mathrm{~ms})$ and onset of choice activity $(202 \mathrm{~ms})$. Shaded regions mark the SO (orange) and SPC (blue) windows. (B-F) Comparison of surface tilt and choice tuning for the example neurons (Figure 3A-E, same order). Black points are surface tilt responses marginalized over slant and distance ( $\mathrm{SO}$ window). In $\mathrm{C}$, the tilt tuning curve has two peaks because the orientation preference changed with distance. Blue points are z-scored choice responses, and the curves are von Mises fits (tuned cases only). (G) Difference between principal surface tilt (SO window) and choice preferences $(\mathrm{N}=166)$. The peak at $0^{\circ}$ indicates that sensory and choice preferences tended to align.

Choice tuning curves with von Mises fits are shown for the example neurons in Figure 5B-F (blue curves). The high (Figures 3A, 5B), intermediate (Figures 3B, 5C), and first low (Figures 3C, 5D) tolerance neurons all had choice activity. The second low tolerance neuron did not have choice activity (Figures 3D, 5E). The neuron with stronger distance than orientation tuning also had choice activity (Figures 3E, 5F). Across the population, the choice tuning curves were well described by von Mises functions (mean $r=0.88 \pm 0.10 \mathrm{SD}, N=201$ ). The mean concentration was $\kappa=3.52 \pm 4.74 \mathrm{SD}$, and the mean half-width at half-height was $52^{\circ} \pm 21^{\circ} \mathrm{SD}$.

For comparison, surface tilt tuning curves marginalized over slant and distance (SO 
window) are shown in Figure 5B-F (black curves). The tilt and choice preferences of the example neurons aligned, even for the neuron with stronger distance than orientation tuning. To quantify this relationship across the population, we used the orientation preferences measured at each distance to compute a principal orientation preference for each neuron (SO window; see Materials and methods). We then compared the principal surface tilt and choice preferences from the von Mises fits (Figure 5G). The median circular difference between the preferences was $-8.3^{\circ}$ and not significantly different from $0^{\circ}$ (circular median test, $p=0.24 ; \mathrm{N}=166$ neurons with orientation and choice tuning), indicating that sensory and choice preferences tended to align.

\section{Changes in 3D selectivity associated with choice activity}

We next tested if choice activity was associated with changes in 3D selectivity. To start, we calculated the correlation between tuning curves in the SO and SPC windows (over all 132 poses). The mean correlation was $r=0.49 \pm 0.27 \mathrm{SD}(\mathrm{N}=437)$, suggesting that tuning was similar in the two time windows but not identical. We hypothesized that the changes reflected a stabilizing effect of choice activity on 3D tuning, but also considered that choice might attenuate selectivity for slant and distance (nuisance variables during tilt discrimination), leaving only selectivity for the taskrelevant variable (tilt) intact.

To illustrate how selectivity changed after the onset of choice activity, the orientation tuning at each distance is shown for the example neurons during the SPC window in Figure 6AE. First consider the three neurons with strong orientation and choice tuning. The selectivity of the neuron with high tolerance in the SO window changed little (Figures 3A, 6A), and its tolerance slightly increased: $\Delta$ Tolerance $=0.08$. Strikingly, the orientation tuning of the neuron with intermediate tolerance in the SO window aligned across distances in the SPC window, matching the tuning at the preferred distance (Figures 3B, 6B), and its tolerance greatly increased: $\Delta$ Tolerance $=0.35$. For the neuron with low tolerance in the SO window, responses at nonpreferred distances remained relatively weak in the SPC window. However, the orientation tuning was now significant at each distance and the preferences were similar (Figures 3C, 6C), resulting in a large increase in tolerance: $\Delta$ Tolerance $=0.46$. Next consider the neuron with low tolerance during the SO window and no choice activity. The selectivity of this neuron changed relatively little during the SPC window (Figures 3D, 6D). Lastly, the distance selective neuron continued to respond most strongly to the furthest distances (Figures 3E, 6E). During the SPC window, 20 neurons (5\%) were distance selective (two-way ANOVA, $p<0.05)$ but not orientation selective $(p$ $\geq 0.05)$, compared to $62(14 \%)$ in the SO window. 


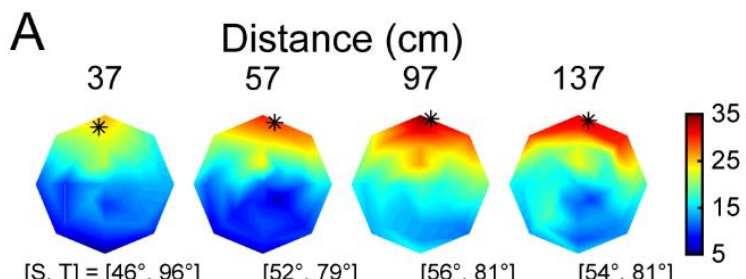

$\mathrm{B}$

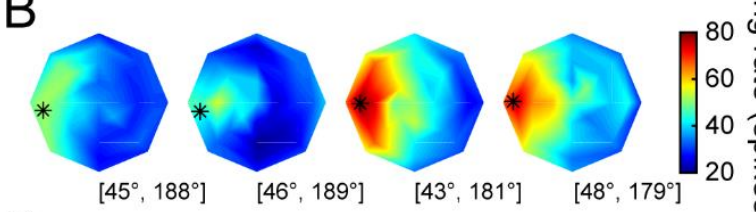

C

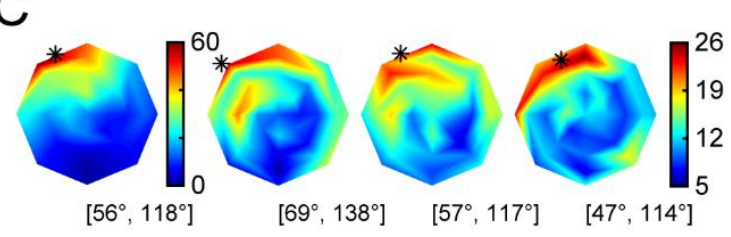

D Distance $(\mathrm{cm})$

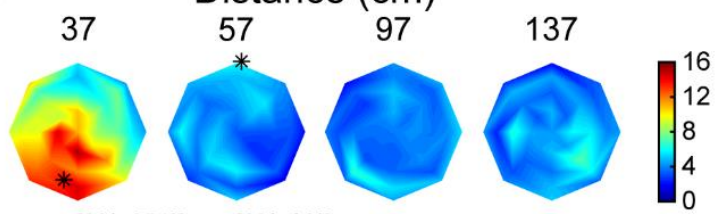

$\left[39^{\circ}, 254^{\circ}\right] \quad\left[63^{\circ}, 86^{\circ}\right]$

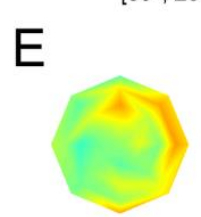

$\mathrm{F}$

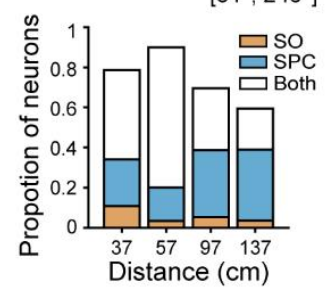

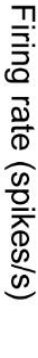

18:289
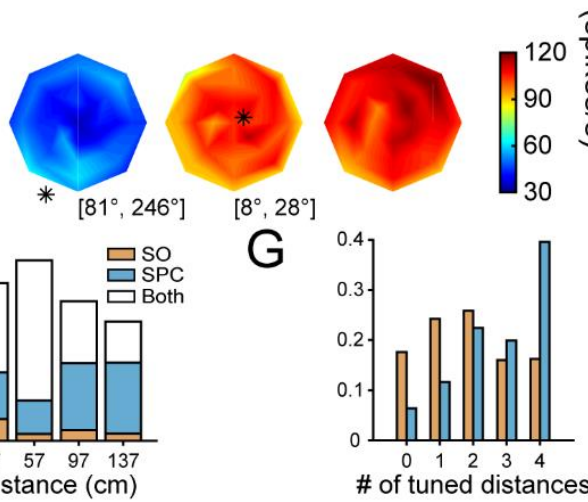

Figure 6. 3D orientation tuning at each distance after the onset of choice activity. (A-E) Responses of the example neurons (Figures 3A-E, 5B-F, same order). (A) Tolerance increased from 0.88 (SO window) to 0.96 (SPC window). (B) Tolerance increased from 0.53 to 0.88 . Note the changes in selectivity at 37 and $57 \mathrm{~cm}$. (C) Tolerance increased from 0.34 to 0.80 . The number of distances with significant orientation tuning increased from one to four. (D) Tolerance increased from 0.24 to 0.33 . (E) Distance selective neuron. (F) Proportion of neurons with orientation tuning at each distance during the SO window only (orange), SPC window only (blue), or both (white). The proportion of neurons with tuning decreased with distance from fixation $(57 \mathrm{~cm})$, consistent with the pattern of behavioral sensitivity (Figure 1E). (G) Proportion of neurons with orientation tuning at each possible number of distances during the SO (orange) and SPC (blue) windows.

Slant and distance tuning persisted in the SPC window for the example neurons, suggesting that choice activity did not attenuate information that was not directly relevant to the task. This finding was typical across the population. For each neuron and time window, we tested for slant and distance tuning (4-way ANOVA with tilt linearized into cosine and sine components, $p<0.05)$. The number of neurons with slant tuning was $334(76 \%)$ in the SO window and 370 $(85 \%)$ in the SPC window. The number of neurons with distance tuning was $414(95 \%)$ in the SO window and $412(94 \%)$ in the SPC window. These results are inconsistent with choice activity attenuating the representation of nuisance variables since the prevalence of slant and distance tuning did not decrease during the SPC window. Instead, choice activity was associated with an increase in the number of neurons with significant orientation tuning (ANOVA, $p<0.05$ ) at each distance (Figure 6F). Indeed, individual neurons often had orientation tuning at two distances during the SO window but all four distances during the SPC window (Figure 6G). 


\section{Choice activity was carried by more tolerant neurons and stabilized 3D selectivity}

We next tested if neurons with robust 3D tuning preferentially carried choice activity. To do so, we compared the SO window tolerances of neurons with and without choice activity (Figure 7A). The mean tolerance was greater for neurons with choice activity (0.61) than without (0.56). Although the difference was not large, it was significant (ANOVA followed by Tukey's HSD test, $p=0.02$ ), indicating that choice activity was preferentially carried by neurons with more robust 3D tuning. We then tested if choice activity further stabilized 3D selectivity by assessing if tolerance increased in the SPC window. The difference in mean tolerance between neurons with (0.72) and without $(0.60)$ choice activity increased in the SPC window (Figure 7B) and was significant $(p=$ $\left.3.8 \times 10^{-9}\right)$. The greater difference was due to an increase in tolerance between time windows for neurons with choice activity $\left(p=3.8 \times 10^{-9}\right.$; Figure 7C). The tolerance of neurons without choice activity did not significantly change $(p=0.06)$. We additionally found that the SPC window tolerance of neurons with choice activity was correlated with the strength of choice activity (log of the tuning curve gain): $r=0.45, p=2.8 \times 10^{-11}$.
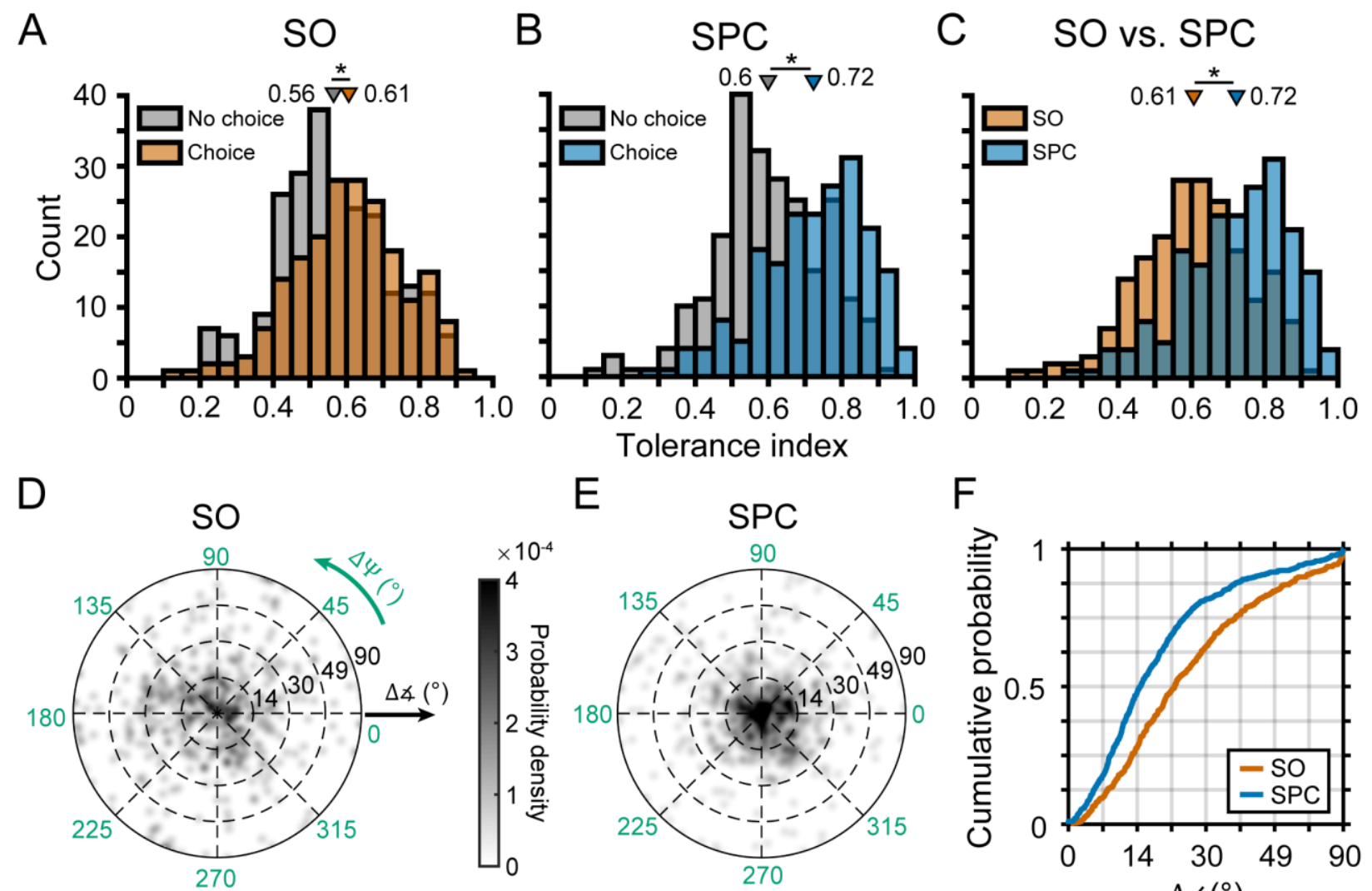

$\mathrm{E}$
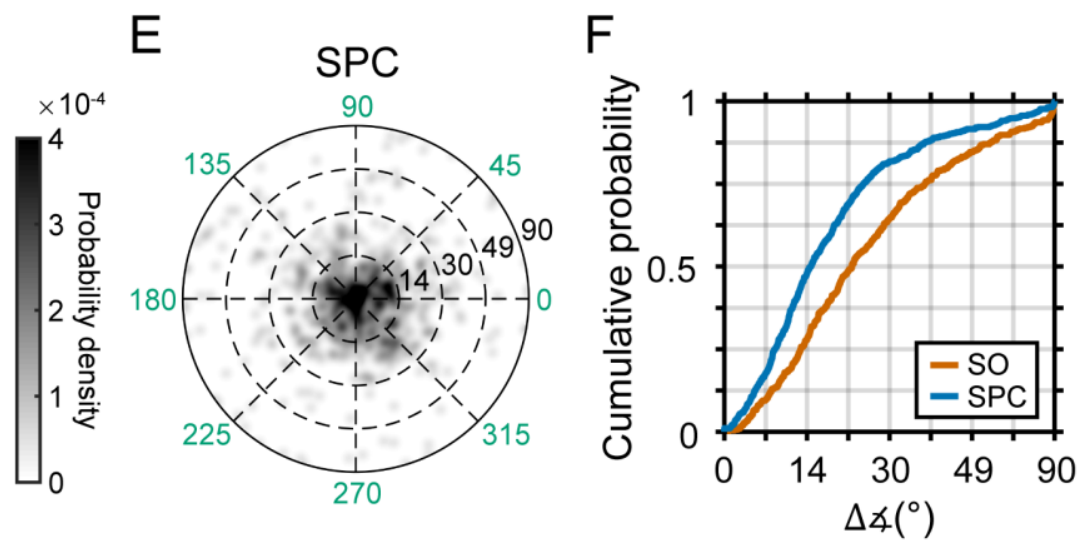

Figure 7. Choice activity was carried by more tolerant neurons and stabilized 3D selectivity. (A) Comparison of tolerance in the SO window for neurons with (orange) and without (gray) choice activity. (B) Comparison of tolerance in the SPC window for neurons with (blue) and without 
choice activity. (C) Comparison of tolerance between the SO and SPC windows for neurons with choice activity. In A-C, triangles mark mean tolerances. Asterisks indicate significant differences. (D) Probability density for the deviations in orientation preference (direction: $\Delta \Psi$; angle: $\Delta \Varangle$ ) from the principal orientation (calculated using both time windows) during the SO window (Gaussian smoothing kernel, $\sigma=0.025$ ). (E) Same as $\mathbf{D}$ but for the SPC window. (F) Cumulative density functions for the angular deviations (radial distance in $\mathbf{D}, \mathbf{E}$ ) in the SO and SPC windows. In $\mathbf{C}-\mathbf{F}$, only neurons with choice activity are included.

To further characterize the effects of choice activity on 3D selectivity, we quantified the differences in orientation preference across distance in each time window. For each neuron with choice activity, we calculated a principal orientation preference using both time windows (see Materials and methods). Deviations in orientation preference from the principal orientation are plotted for each time window in Figure 7D,E. In these plots, the angular variable is the direction that the preferred orientation at a given distance deviated from the principal orientation $(\Delta \Psi)$, and the radial variable is the angular deviation $(\Delta x)$. The origin indicates no difference, and points on the outer ring indicate the maximal difference $\left(90^{\circ}\right)$. The deviations were not significantly different from the origin in either time window (test for a specified principal axis; both $p$-values $\geq 0.09$ ) (Fisher et al., 1993). To assess how much the orientation preferences deviated from the principal orientation, we calculated cumulative density functions for the angular deviations (Figure 7F). The mean deviation was greater in the SO $\left(28.3^{\circ}\right)$ than the SPC $\left(20.4^{\circ}\right)$ window, and the cumulative densities significantly differed (Kolmogorov-Smirnov test, $p=1.4 \times 10^{-10}$ ). Thus, the orientation preferences became more similar across distance after the onset of choice activity. We repeated this analysis for neurons without choice activity, and found that the cumulative densities for the two time windows were not significantly different $(p=0.91)$. Thus, 3D selectivity stabilized in the SPC window, but only for neurons with choice activity.

\section{Sensorimotor associations in CIP are mediated by choice activity}

We lastly conducted the first test of motor-related activity in CIP. Anatomical and effective connectivity studies indicate that CIP receives (direct or indirect) input from V3A and projects to LIP (Nakamura et al., 2001; Premereur et al., 2015; Van Dromme et al., 2016), two areas with saccade-related activity (Andersen et al., 1992; Nakamura and Colby, 2000). We therefore tested for saccade direction tuning using a visually guided saccade task (Munoz and Wurtz, 1995; Hanes and Schall, 1996) (Figure 8A).

We computed eight population-level time courses of saccade-related activity, relative to the saccade direction that elicited the maximum response for each neuron (Figure 8B). The time course of saccade-related activity had parametric tuning, with an amplitude that symmetrically 
decreased with greater deviation from the preferred direction. Significant saccade direction selectivity began $102 \mathrm{~ms}$ before saccade onset (ANOVA, $p<0.05$ ). We further found that the activity predicted the saccade timing. For each neuron, we labeled every trial in which a saccade was made in the preferred direction according to the saccade latency in quartiles. Time courses were then calculated for each quartile (Figure 8C; inset shows the latency histogram). The four curves approximately intersected 15 ms before saccade onset, suggestive of a saccade initiation threshold ( 42 spikes/s, black circle). Moreover, the activity increased more slowly when the saccade latency was longer (colored circles mark when each curve significantly deviated from baseline; ANOVA, $p<0.05)$. For each curve, we computed the growth rate (linear slope) between the start of activity and the putative saccade threshold. Consistent with frontal eye field findings (Hanes and Schall, 1996), there was an inverse linear relationship between the growth rate and mean saccade latency (Figure 8D). Thus, saccade-related activity in CIP functionally correlated with both the saccade direction and timing during the visually guided saccade task.
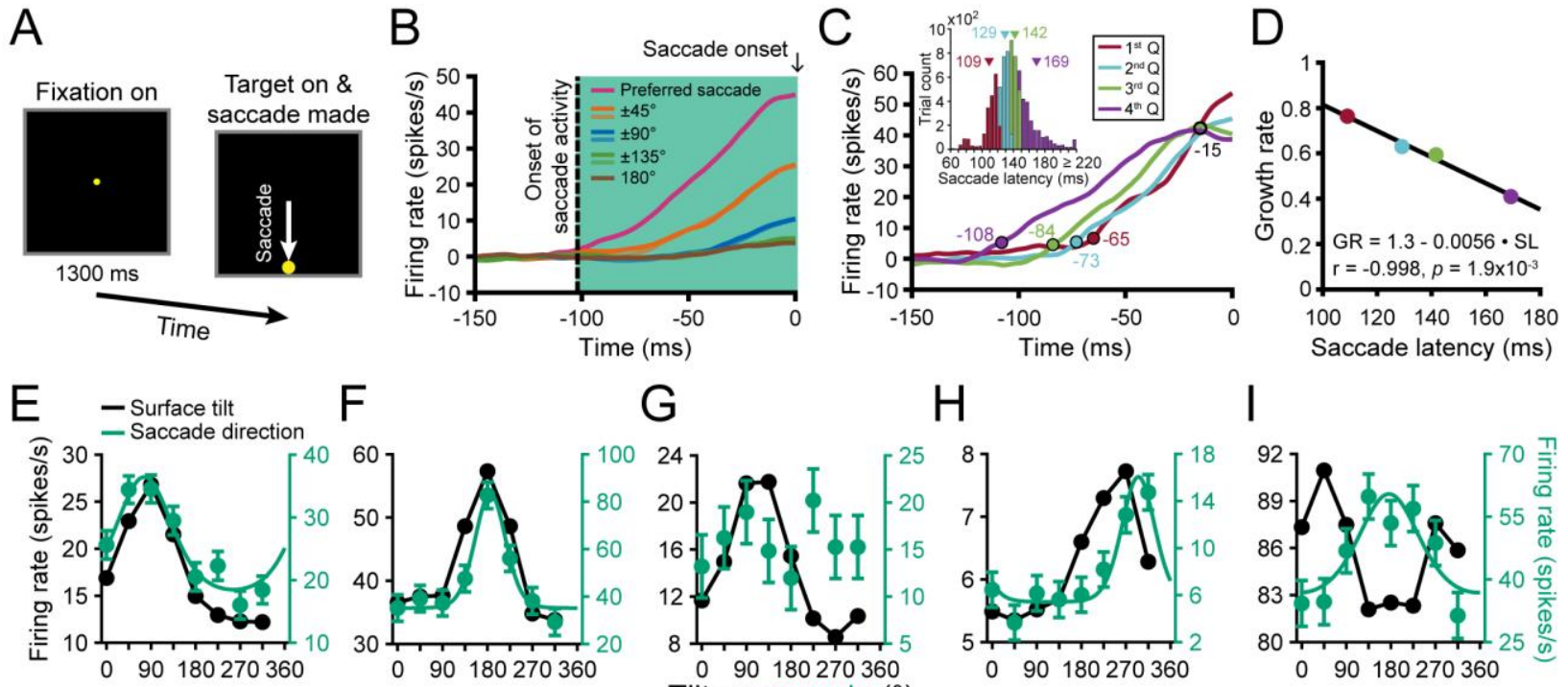

Tilt or saccade $\left({ }^{\circ}\right)$
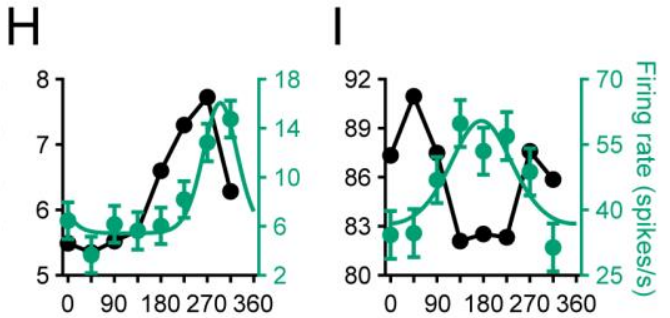

Figure 8. Saccade-related activity and sensorimotor associations. (A) Saccade task. After fixating a target at the center of the screen for $1.3 \mathrm{~s}$, the fixation target disappeared and a saccade target appeared at one of eight locations coinciding with the choice targets in the discrimination task. (B) Time course of saccade-related activity. Curves are average responses for each of the eight saccade directions relative to the preferred direction. Saccade onset $=0 \mathrm{~ms}$. Vertical dashed line marks the onset of saccade activity $(-102 \mathrm{~ms})$. Shaded region marks the window used to assess saccade activity. (C) Time course of saccade-related activity depending on the saccade latency in quartiles (Q). Colored circles mark the start of activity. Open black circle marks a putative saccade threshold. The times are indicated next to each circle. Inset shows the histogram of saccade latencies with quartiles colored. Triangles mark mean latencies. (D) Inverse linear relationship between the growth rate (GR) of saccade-related activity and mean saccade latency $(\mathrm{SL})$ across quartiles. (E-I) Comparison of surface tilt and saccade direction tuning curves for the example neurons (Figures 3A-E, 5B-F, 6A-E, same order). Black points are surface tilt responses 
446

447

marginalized over slant and distance (SO+SPC windows). Green points are saccade direction responses, and the curves are von Mises fits (tuned cases only).

Across the population, 274 neurons ( 63\%) had saccade direction tuning (ANOVA, $p<$ 0.05). The tuning curves were well described by von Mises functions (mean $r=0.91 \pm 0.10 \mathrm{SD}$ ). The mean concentration parameter was $\kappa=4.67 \pm 4.84 \mathrm{SD}$, and the mean half-width at halfheight was $42^{\circ} \pm 17^{\circ} \mathrm{SD}$. Saccade direction tuning curves with von Mises fits are shown for the example neurons in Figure 8E-I (green curves). The first two neurons had orientation (Figures 3A,B, 6A,B) and choice (Figure 5B,C) tuning, as well as saccade direction tuning (Figure 8E,F). The third neuron had orientation (Figures 3C, 6C) and choice (Figure 5D) tuning, but not saccade direction tuning (Figure 8G). The fourth neuron, which had orientation (Figures 3D, 6D) but not choice (Figure 5E) tuning, had saccade direction tuning (Figure $8 \mathrm{H}$ ). Lastly, the neuron with stronger distance than orientation tuning (Figures 3E, 6E) had both choice (Figure 5F) and saccade direction (Figure 8I) tuning, but the choice and saccade preferences were not aligned. These various differences illustrate that choice and saccade response properties were dissociable.

For comparison, surface tilt tuning curves marginalized over slant and distance (SO+SPC windows) are shown in Figure 8E-I (black curves). The tilt and saccade direction preferences of the example neurons with strong orientation and choice tuning were well aligned (Figure 8E,F), implying a sensorimotor association at the individual neuron level. The preferences were also reasonably well aligned for the neuron with strong orientation but not choice tuning (Figure $\mathbf{8 H}$ ). They were not aligned for the neuron with stronger distance than orientation tuning (Figure 8I). To quantify the sensorimotor association, we compared the principal tilt (SO+SPC windows) and saccade direction preferences from the von Mises fits. Across the population, the preferences aligned both for neurons without (Figure 9A) and with (Figure 9B) choice activity. For neurons without (with) choice activity, the median circular difference between the preferences was $-0.9^{\circ}$ $\left(-1.6^{\circ}\right)$ and not significantly different from $0^{\circ}$ (circular median test, both $p$-values $\geq 0.61$ ). Although the preferences tended to align regardless of choice activity, the distribution was significantly wider for neurons without (circular variance $=0.74$ ) than with $(0.36)$ choice activity (two-sample concentration difference test, $p=3.2 \times 10^{-5}$ ) (Fisher, 1995), indicating that the sensorimotor association was strongest for neurons with choice activity. 

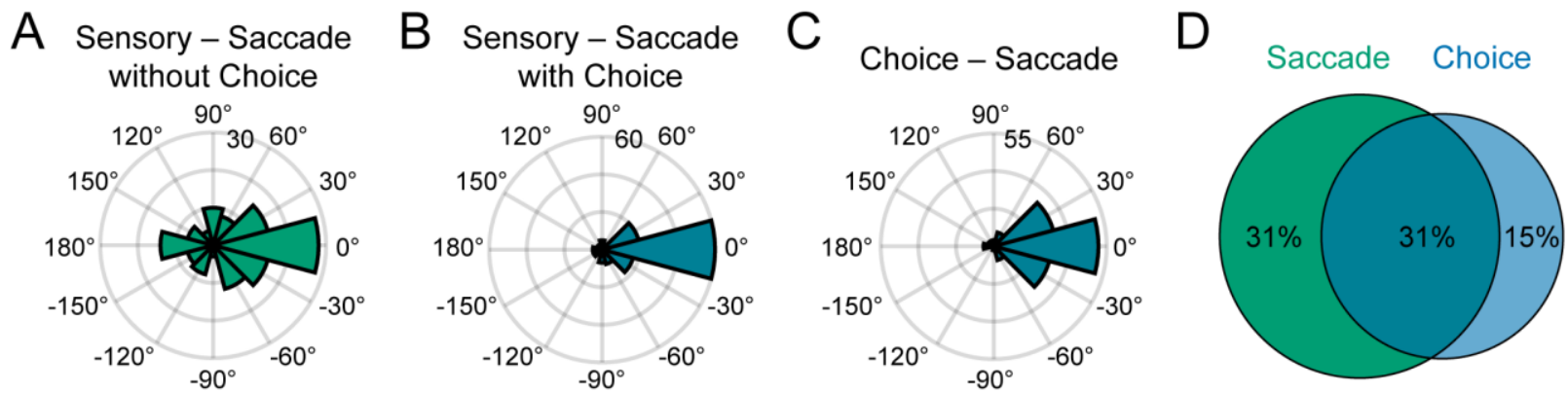

Figure 9. Sensorimotor associations were mediated by choice activity. (A) Differences between principal surface tilts and preferred saccade directions for neurons without choice activity ( $\mathrm{N}=$ 131). (B) Same as $\mathbf{A}$ but for neurons with choice activity $(N=135)$. (C) Differences between choice and saccade direction preferences $(\mathrm{N}=137)$. (D) Venn diagram showing the proportion of neurons with saccade activity only (green), choice activity only (blue), and both (teal).

Across the population, 137 neurons (31\%) had both choice and saccade direction tuning. The median circular difference between the preferences was $2.9^{\circ}$ and not significantly different from $0^{\circ}$ (circular median test, $p=0.61$ ), indicating that the preferences tended to align (Figure 9C). There were also many neurons with saccade activity only $(137,31 \%)$ or choice activity only (64, 15\%), further indicating that saccade and choice responses were dissociable (Figure 9D).

\section{Discussion}

Interacting with the environment requires the creation of robust representations of 3D information that the epithelia cannot directly sense, and mapping those representations to motor responses. We investigated the relationships between the quality of 3D representations, choice-related activity, and motor-related activity in CIP. The behavioral task required the monkeys to report the tilt of a plane, regardless of its slant or distance. As such, slant and distance were nuisance variables that were not of direct interest but modulated task performance. Rather than attenuating selectivity for the nuisance variables, choice activity improved the fidelity of the 3D representation. Thus, the low-dimensional task (report the tilt) did not reduce the dimensionality of the neuronal representation of the multi-dimensional (tilt, slant, and distance) stimulus. This may enable more robust 3D perception and flexible processing. For example, if choice activity attenuated selectivity for features not directly relevant to the task at hand, performance would be impaired if the task and relevant information unexpectedly changed.

The monkeys were trained to report which side of a plane was nearest, whereas a previous CIP study had monkeys report which side was furthest (Elmore et al., 2019). In both studies, surface tilt and choice preferences aligned. For example, given a neuron preferring a bottom-near tilt, the preferred choice report tended to be the lower target in this study but the upper target in 
507 the previous study. Here, we further found that many CIP neurons have saccade-related activity,

508 that saccade direction preferences aligned with the sensory and choice preferences, and that

509 choice- and saccade-related activities were dissociable. These findings together reveal a flexible,

510 experience-dependent mapping between sensory, choice-, and motor-related activity, indicating

511 that experience has ongoing effects on the functional properties of CIP neurons, as occurs in

512 downstream area LIP (Freedman and Assad, 2006; Law and Gold, 2008; Bennur and Gold, 2011).

\section{Building selectivity for 3D object pose}

515 Transforming 2D retinal images into 3D representations is a nonlinear optimization problem 516 (Hartley and Zisserman, 2003). Neurons in CIP achieve 3D selectivity by integrating stereoscopic 517 and perspective cues (Tsutsui et al., 2001; Tsutsui et al., 2002; Rosenberg and Angelaki, 2014b).

518 A stereoscopic representation of 3D orientation that is tolerant to distance requires an encoding 519 of relative disparity gradients that takes into account the nonlinear relationship between depth and disparity. We found that the orientation selectivity of many CIP neurons was highly tolerant to distance. That tolerance cannot simply reflect tuning for perspective cues (even though they were independent of distance) because the vast majority of neurons had significant distance tuning. Our findings thus reveal a high-level representation of 3D object pose created using relative disparity computations. How might this representation be achieved?

Functional properties and connectivity data suggest that tuning for 3D object pose is built hierarchically. Neurons in V1 represent local absolute disparities (Cumming and Parker, 1997, 1999, 2000). A transformation from absolute to relative disparity selectivity likely proceeds in V2

528 (Thomas et al., 2002) and V3A where absolute disparity signals may still predominate (Anzai et 529 al., 2011) but the representation of disparity gradients may begin (Elmore et al., 2019). The 2D530 to-3D transformation likely progresses further in the posterior intraparietal area where selectivity 531 for local relative disparity gradients may arise (Alizadeh et al., 2018), ultimately achieving 3D 532 object pose tuning in CIP.

533 Recent studies identified brain regions that represent the 3D structure of the environment 534 (Vaziri et al., 2014; Lescroart and Gallant, 2019). How 3D object and environment representations 535 interact at the neuronal level is currently unknown. To characterize sensory and sensorimotor 536 processing under more ecologically realistic contexts, it will be useful to study how CIP represents 537 the pose of more naturalistic objects with which the animals interact, and if that representation is 538 shaped by the surrounding environment. 


\section{Separable orientation and distance tuning}

541 Distinguishing 3D representations from lower-level feature selectivity is a longstanding problem

542 (Janssen et al., 2000; Nguyenkim and DeAngelis, 2003; Alizadeh et al., 2018; Elmore et al., 2019).

543 Showing that 3D orientation selectivity is relatively invariant to distance when the fixation distance

544 is held constant is a common criterion to conclude 3D tuning. This fundamental test was never

545 previously performed in CIP. We found that slant preferences tended to slightly increase with

546 greater distance behind fixation. Although the increase was small, the finding indicates that

547 orientation and distance tuning was not strictly separable. The behavioral data likewise showed

548 that the monkeys' sensitivity fell off more slowly with distance from fixation at larger compared to

549 smaller slants. This behavioral finding implies that larger slants were required to elicit robust

550 stimulus-selective neuronal responses at distances further from fixation, predicting the observed

551 increase in slant preference and suggesting that strictly separable tuning may not be a reasonable

552 expectation. It is unlikely that this conclusion rests upon using stimuli with fixed slants as opposed

553 to fixed disparity gradients. Although the disparity gradient signaling a given slant is distance

554 dependent (due to the nonlinear relationship between depth and disparity), using fixed disparity

555 gradients would mean that the 3D shape of the stimuli would change with distance. As such,

556 regardless of whether fixed slants or disparity gradients are used, it is unlikely that tuning will be

557 strictly separable over orientation and distance.

558

\section{Potential origins of choice-related activity}

560 Choice activity is traditionally associated with feedforward contributions to perception (Celebrini 561 and Newsome, 1994; Britten et al., 1996; Dodd et al., 2001; Nienborg and Cumming, 2006; Gu et 562 al., 2007). This possibility has been questioned on the basis that choice activity, attentional effects, 563 and correlated activity can be conflated (Cumming and Nienborg, 2016). Existing choice activity 564 measures likely reflect combinations of these factors (Haefner et al., 2013; Gu et al., 2014). We 565 found that surface tilt and choice preferences typically aligned. This is expected for a feedforward 566 origin of choice activity, and contrasts with the potentially non-specific effects of a feedback origin, 567 such as proposed for the ventral intraparietal area (Zaidel et al., 2017). We further found that 568 choice activity was preferentially carried by neurons which robustly represented the task-relevant 569 sensory information, and that the strength of choice activity increased with sensory tolerance. The 570 extent to which subcortical vestibular neurons resolve the gravito-inertial acceleration ambiguity 571 necessary to represent translation independent of head tilt (Dakin and Rosenberg, 2018) similarly 572 correlates with the strength of choice activity (Liu et al., 2013). Across modalities, these results 573 reveal that neurons which have resolved fundamental ambiguities about the sensory information 
574 that an animal is actively discriminating preferentially carry choice activity. This may reflect a

575 feedforward origin of choice activity, such that neurons with more robust tuning for task-relevant

576 information have greater weight in the neural readout. Indeed, decoding neurons that are more

577 tolerant to nuisance variables may simplify readout since marginalizing out non-relevant features

578 would be more straightforward.

579

580

\section{Sensorimotor associations}

581 We discovered two roles for CIP beyond sensory processing. First, we showed that CIP activity 582 functionally correlates with both the direction and timing of visually guided saccades, implicating 583 the area in visuomotor control for the first time. Second, we found sensorimotor associations at 584 the single neuron level, which were strongest for neurons with choice activity (i.e., those with more 585 robust 3D selectivity). This finding may reflect that neurons with robust sensory representations 586 and stronger sensorimotor associations have greater weight in determining motor responses. 587 Associating robust sensory and motor activity at the single neuron level may help ensure 588 successful and timely interactions with the environment since the same neurons representing the 589 relevant sensory information also signal the appropriate motor response. While our findings 590 implicate CIP in visuomotor functioning, the area is also connected with prehensile areas, 591 suggesting that its sensorimotor functions may be more general. This possibility is consistent with 592 recent findings implicating human parietal regions that integrate visual orientation and saccade 593 signals in the updating of grasp plans during eye movements (Baltaretu et al., 2020).

These findings reveal previously unrecognized roles for choice activity in improving the 595 fidelity of high-level sensory representations and mediating sensorimotor associations, and show 596 that CIP achieves a complete and flexible sensorimotor association for transforming information 597 about the 3D world into overt behavior.

\section{Materials and methods}

600 Animal preparation

601 All surgeries and experimental procedures were approved by the Institutional Animal Care and 602 Use Committee at the University of Wisconsin-Madison, and in accordance with NIH guidelines. 603 Two male rhesus monkeys (Macaca mulatta; Monkey L: 6 years of age, $9.5 \mathrm{~kg}$; Monkey F: 5 604 years, $\sim 6.4 \mathrm{~kg}$ ) were surgically implanted with a Delrin ring for head restraint and attaching a 605 removable recording grid for guiding electrodes (Rosenberg et al., 2013). After recovery, the 606 monkeys were trained to sit in a primate chair with head restraint, and to fixate visual targets 607 within $2^{\circ}$ version and $1^{\circ}$ vergence windows. Vergence was enforced during the experiments, 
608

609

610

611

612

613

614

615

616

617

618

619

620

621

622

623

624

625

626

627

628

629

630

631

632

633

634

635

636

637

638

639

640

641

which is often not the case in 3D vision studies (e.g., Sanada and DeAngelis, 2014), and was also factored into the analyses using ANCOVAs to test for main effects of stimulus tuning with vergence included as a covariate (DeAngelis and Uka, 2003).

\section{Experimental control and stimulus presentation}

Experimental control was performed using the open-source REC-GUI software (Kim et al., 2019). Stimuli were rendered using Psychtoolbox 3 (MATLAB R2016b; NVIDIA GeForce GTX 970). They were rear-projected onto a polarization preserving screen (Stewart Film Screen, Inc.) using a DLP LED projector (PROPixx; VPixx Technologies, Inc.) with 1,280 x 720 pixel resolution $\left(70^{\circ} \times 43^{\circ}\right)$ at $240 \mathrm{~Hz}(120 \mathrm{~Hz} / \mathrm{eye})$. The screen was positioned $57 \mathrm{~cm}$ from the monkey. Polarized glasses were worn. A phototransistor circuit was used to confirm the synchronization of left and right eye images, and to align neuronal responses to the stimulus onset. Eye positions were monitored optically at $1 \mathrm{KHz}$ (EyeLink 1000 plus, SR Research).

\section{Visual stimuli}

The stimuli were the same as the combined-cue stimuli in our previous work (Chang et al., 2020). They consisted of 250 nonoverlapping dots uniformly distributed across a plane. The stimulus envelope was a $20^{\circ}$ diameter circle on the screen. The background was gray $\left(11.06 \mathrm{~cd} / \mathrm{m}^{2}\right)$ and the dots were bright $\left(35.1 \mathrm{~cd} / \mathrm{m}^{2}\right)$, measured through the glasses (PR-524 LiteMate, Photo Research). Planes were presented at all combinations of eight tilts ( $0^{\circ}$ to $315^{\circ}, 45^{\circ}$ steps), five slants $\left(0^{\circ}\right.$ to $60^{\circ}, 15^{\circ}$ steps $)$, and four distances $(37,57,97$, and $137 \mathrm{~cm})$. Fixation was always at screen distance $(57 \mathrm{~cm})$. At $37 \mathrm{~cm}$, all dots were in front of the fixation target. At $57 \mathrm{~cm}$, the dots were distributed about the fixation target. At 97 and $137 \mathrm{~cm}$, all dots were behind fixation. Presenting the stimuli in front of, distributed about, and behind fixation prevented the monkeys from relying on local absolute disparity cues to perform the task. The dots were rendered with stereoscopic and perspective cues, and scaled according to the distance so that their screen size only depended on the slant. The baseline dot size was $0.35^{\circ}$ isotropic.

\section{Tilt discrimination task}

The monkeys were trained to perform an 8AFC tilt discrimination task (Chang et al., 2020). Each trial began with fixation of a circular target $\left(0.3^{\circ}\right)$ at the center of the screen for $300 \mathrm{~ms}$. A plane then appeared at the center of the screen for $1 \mathrm{~s}$. The target and plane then disappeared, and 8 choice targets appeared at polar angles of $0^{\circ}$ to $315^{\circ}$ in $45^{\circ}$ steps $\left(11^{\circ}\right.$ eccentricity). The nearest side of the plane was reported by making a saccade to the corresponding target for a liquid reward. 
642 Responses to frontoparallel planes (slant $=0^{\circ}$, tilt undefined) were rewarded with equal probability

$643(12.5 \%)$.

644

645

\section{Visually guided saccade task}

646 Each trial began with fixation of a target at the center of the screen for $1.3 \mathrm{~s}$. The fixation target 647 then disappeared and a saccade target appeared at one of the choice target locations. A saccade 648 to the target was made for a liquid reward.

649

650

\section{Experimental protocol}

651 Stimuli were presented in a pseudorandom order within a block design. A block included one 652 completed trial for each of the following: ( $I$ ) planes: (8 tilts $\times 4$ non-zero slants +8 frontoparallel 653 trials) $\times 4$ distances $(\mathrm{N}=160)$, and (ii) saccades: 8 directions $\times 4$ repeats $(\mathrm{N}=32)$. A trial was 654 aborted and data discarded if fixation was broken before the choice or saccade targets appeared, 655 or if the trial was not completed within 500 ms of their appearance. At least five complete blocks 656 were required to include a neuron for analysis.

657

658

\section{Neuronal recordings}

659 Area CIP was identified based on anatomical and functional properties (Rosenberg et al., 2013; 660 Rosenberg and Angelaki, 2014b, a; Elmore et al., 2019). Briefly, the CARET software was used 661 to register the structural MRIs to the F99 atlas (Van Essen et al., 2001) and align the recording 662 grids to estimate electrode trajectories. The area was functionally distinguished from neighboring 663 regions based on 3D orientation selectivity and large receptive fields. Recordings were performed 664 using silicone linear array probes with four or eight tetrodes (NeuroNexus, Inc.). Tetrodes were 665 separated by $300 \mu \mathrm{m}$. Electrodes within a tetrode were arranged in a diamond pattern and 666 separated by $25 \mu \mathrm{m}$. Neuronal signals were sampled at $30 \mathrm{KHz}$ and stored with eye movement 667 and phototransistor traces sampled at $1 \mathrm{KHz}$ (Scout Processor; Ripple, Inc.). Tetrode-based spike 668 sorting was performed offline using the KlustaKwik (K. Harris) semi-automatic clustering algorithm 669 in MClust (MClust-4.0, A.D. Redish et al.) followed by manual refinement using Offline Sorter 670 (Plexon, Inc.). Only well-isolated single neurons verified by at least two authors were included for 671 analysis: 214 neurons from the left hemisphere of Monkey $L$ (26 sessions) and 209 neurons from 672 the right hemisphere of Monkey F (27 sessions).

673

\section{Analysis of behavioral data}

675 Tilt discrimination performance was quantified by fitting a von Mises probability density function 
676

677

678

679

680

681

682

683

684

685

686

687

688

689

690

691

692

693

694

695

696

697

698

699

700

701

702

703

704

705

706

707

708

709

710

to the distribution of reported tilt errors (Chang et al., 2020), $\Delta$ Tilt $=$ reported tilt - presented tilt:

$$
V M(\Delta \mathrm{Tilt})=e^{\kappa \cdot \cos (\Delta \mathrm{Tilt}-\mu)} /\left(2 \pi \cdot I_{0}(\kappa)\right)
$$

The mean $(\mu)$ and concentration $(\kappa)$ parameters describe accuracy and sensitivity, respectively (Seilheimer et al., 2014; Dakin and Rosenberg, 2018). Values of $\mu$ closer to 0 indicate greater accuracy. Larger $\kappa$ indicate greater sensitivity. Given the $45^{\circ}$ tilt sampling interval, we set $\kappa=18$ as the upper bound in the estimation routine (Chang et al., 2020). A modified Bessel function of order $0, I_{0}(\kappa)$, normalizes the function to have unit area.

\section{Analyses of neuronal data}

Visual response latency. To estimate a neuron's visual response latency, a spike density function (SDF) was created for each trial that a plane was presented by convolving the spike train (1 ms bins, aligned to the stimulus onset) with the function (Schwemmer et al., 2015):

$$
\alpha(t)=\alpha^{*} \cdot H(t) \cdot\left[e^{-\tau_{d} \cdot t}-e^{-\tau_{r} \cdot t}\right]
$$

Here, $\alpha^{*}$ normalizes $\alpha(t)$ to have unit area, $H(t)$ is the Heaviside function, and $\tau_{d}=0.05$ and $\tau_{r}=1.05$ are time constants. The response latency was defined as the first time point after stimulus onset that the firing rate was significantly different from baseline for at least $30 \mathrm{~ms}$ (ANOVA, $p<0.05$ ). The baseline firing rate was calculated using the last $150 \mathrm{~ms}$ of the fixation periods preceding the stimulus onsets.

Choice-related activity. We tested for choice-related activity using the frontoparallel plane data. To combine data across distances, we z-scored all baseline subtracted frontoparallel plane responses at each distance. We then grouped the $z$-scored firing rates according to the choice. A neuron was classified as having choice activity if the z-scored firing rates significantly depended on the choice (ANOVA, $p<0.05$ ). These neurons were used to estimate the time course of choice activity. Average SDFs were calculated for each choice (aligned to the stimulus onset), and labeled relative to the choice that elicited the maximum $z$-scored firing rate for the neuron: preferred choice, $\pm 45^{\circ}, \pm 90^{\circ}, \pm 135^{\circ}$, and $180^{\circ}$. The SDFs were averaged across neurons to create eight population-level time courses. The onset of choice activity was defined as the first time point after stimulus onset that the time courses significantly differed (ANOVA, $p<0.05$ ). To refine the estimate, we iteratively repeated this process with all neurons, each time calculating the firing rates starting from the previous estimate of the onset of choice activity to the end of the $1 \mathrm{~s}$ stimulus presentation (firing rates were first calculated using the full $1 \mathrm{~s}$ ). This process was 
711

712

713

714

715

716

717

718

719

720

721

722

723

724

725

726

727

728

729

730

731

732

733

734

735

736

737

738

739

740

741

742

743

repeated until the onset no longer changed.

Quantifying orientation selectivity. For each distance and time window, we tested for orientation tuning (ANOVA, $p<0.05$, with Bonferroni-Holm correction). The preferred tilt and slant was estimated for each significant case by fitting a Bingham function (Rosenberg et al., 2013). Differences in orientation preference were assessed as follows. First, we calculated the principal orientation about which the measured orientation preferences clustered (Fisher et al., 1993). For each distance and time window with significant tuning $(\mathrm{N} \leq 8)$, the surface normal vector $n_{i}=$ $\left[\begin{array}{lll}x_{i} & y_{i} & z_{i}\end{array}\right]^{T}$ of the plane with the preferred tilt $\left(T_{i}\right)$ and slant $\left(S_{i}\right)$ was calculated:

$$
\begin{gathered}
x_{i}=\cos \left(T_{i}\right) \cdot \sin \left(S_{i}\right), \\
y_{i}=\sin \left(T_{i}\right) \cdot \sin \left(S_{i}\right), \\
z_{i}=\cos \left(S_{i}\right) .
\end{gathered}
$$

(equation 3)

The normal vectors were then arranged in a matrix and the eigenvectors calculated. The principal orientation was defined as the eigenvector with the greatest eigenvalue. Principal orientations were calculated using the SO window only and both (SO+SPC) windows. Second, we rotated the normal vectors such that the principal orientation aligned with the north pole $(n=$ $\left.\left[\begin{array}{lll}0 & 0 & 1\end{array}\right]^{T}\right)$ :

$$
n_{i}^{\prime}=r T\left(-T_{p}\right) \cdot r S\left(S_{p}\right) \cdot r T\left(T_{p}\right) \cdot n_{i}
$$

Here, $n_{i}^{\prime}$ is a rotated normal, $T_{p}$ and $S_{p}$ are the tilt and slant of the principal orientation, respectively, and $r T$ and $r S$ are rotation matrices:

$$
r T\left(T_{p}\right)=\left[\begin{array}{ccc}
\cos \left(T_{p}\right) & \sin \left(T_{p}\right) & 0 \\
-\sin \left(T_{p}\right) & \cos \left(T_{p}\right) & 0 \\
0 & 0 & 1
\end{array}\right]
$$

and

$$
r S\left(S_{p}\right)=\left[\begin{array}{ccc}
\cos \left(S_{p}\right) & 0 & -\sin \left(S_{p}\right) \\
0 & 1 & 0 \\
\sin \left(S_{p}\right) & 0 & \cos \left(S_{p}\right)
\end{array}\right]
$$

The equatorial projection of the rotated normal vectors is a standardized polar coordinate space that describes deviations in orientation preference.

Quantifying tolerance. To quantify how tolerant each neuron's orientation selectivity was to distance, we fit the $33 \times 4$ (orientations $x$ distances) matrix of responses with a multiplicatively separable model:

$$
R(\theta, D)=D C+g \cdot H(\theta) \cdot F(D)
$$


Here, $R(\theta, D)$ is the response to orientation $\theta$ (tilt and slant) and distance $D, D C$ is an offset, $g$ is the response gain, $H(\theta)$ is the orientation tuning, and $F(D)$ is the distance tuning. Fitting was performed using singular value decomposition within a minimization routine to find the $D C, g$ (first singular value), and $H$ and $F$ (first pair of singular vectors) that minimized the Euclidean norm of the error. A tolerance index was defined as the average Pearson correlation between the observed and model orientation tuning curves at each distance. A value of 1 indicates that the shape of the orientation tuning curve was perfectly invariant to distance. Values closer to 0 imply sensitivity to lower-level visual features.

We also tested an additively separable model, $R(\theta, D)=D C+H(\theta)+F(D)$. To fit the model, we constructed a system of equations for the 132 orientation and distance combinations. The closed form solution for the resulting linear regression problem was found by including a regularization parameter that minimized the Euclidian norm of the error (Hastie et al., 2009), giving this model one more free parameter than the multiplicatively separable model. Results with the additive model are not presented because the responses of every neuron in both time windows (846 comparisons) were better described by the multiplicatively separable model.

Saccade analysis. A saccade onset was defined as the first time point that the velocity of either eye was $\geq 150 \%$ s. A neuron was classified as having saccade direction selectivity if the baseline subtracted firing rates significantly depended on the saccade direction (ANOVA, $p<$ 0.05). These neurons were used to estimate the time course of saccade activity. Average SDFs were calculated for each saccade direction (aligned to the saccade onset), and labeled relative to the saccade direction that elicited the maximum firing rate for the neuron. The SDFs were averaged across neurons to create eight time courses. The onset of saccade activity was defined as the earliest time point before saccade onset that the time courses significantly differed (ANOVA, $p<0.05)$. To refine the estimate, we iteratively repeated this process with all neurons, each time calculating the firing rates from the previous estimate of the onset of saccade activity to the start of the saccade. This process was repeated until the onset no longer changed.

\section{Acknowledgments}

We thank Satchal Postlewaite for help with spike sorting. This work was supported by the Alfred P. Sloan Foundation (FG-2016-6468), Whitehall Foundation (2016-08-18), Greater Milwaukee Foundation (Shaw Scientist Award), and the National Institutes of Health (EY029438). Further support was provided by National Institutes of Health Grant P51OD011106 to the Wisconsin National Primate Research Center, University of Wisconsin-Madison.

\section{Competing Interests}

The authors declare that no competing interests exist. 
817

818

819

820

821

822

823

824

825

826

827

828

829

830

831

\section{References}

Alizadeh AM, Van Dromme I, Verhoef BE, Janssen P (2018) Caudal intraparietal sulcus and three-dimensional vision: A combined functional magnetic resonance imaging and single-cell study. Neuroimage 166:46-59.

Andersen RA, Brotchie PR, Mazzoni P (1992) Evidence for the lateral intraparietal area as the parietal eye field. Curr Opin Neurobiol 2:840-846.

Anzai A, Chowdhury SA, DeAngelis GC (2011) Coding of stereoscopic depth information in visual areas V3 and V3A. J Neurosci 31:10270-10282.

Baltaretu BR, Monaco S, Velji-lbrahim J, Luabeya GN, Crawford JD (2020) Parietal cortex integrates saccade and object orientation signals to update grasp plans. J Neurosci.

Baumann MA, Fluet MC, Scherberger H (2009) Context-specific grasp movement representation in the macaque anterior intraparietal area. J Neurosci 29:6436-6448.

Bennur S, Gold JI (2011) Distinct representations of a perceptual decision and the associated oculomotor plan in the monkey lateral intraparietal area. J Neurosci 31:913-921.

Breveglieri R, Bosco A, Galletti C, Passarelli L, Fattori P (2016) Neural activity in the medial parietal area V6A while grasping with or without visual feedback. Sci Rep 6:28893.

Britten KH, Newsome WT, Shadlen MN, Celebrini S, Movshon JA (1996) A relationship between behavioral choice and the visual responses of neurons in macaque MT. Vis Neurosci 13:87100.

Brovelli A, Ding M, Ledberg A, Chen Y, Nakamura R, Bressler SL (2004) Beta oscillations in a large-scale sensorimotor cortical network: Directional influences revealed by Granger causality. Proc Natl Acad Sci U S A 101:9849-9854.

Buneo CA, Andersen RA (2006) The posterior parietal cortex: Sensorimotor interface for the planning and online control of visually guided movements. Neuropsychologia 44:2594-2606.

Buneo CA, Jarvis MR, Batista AP, Andersen RA (2002) Direct visuomotor transformations for reaching. Nature 416:632-636.

Celebrini S, Newsome WT (1994) Neuronal and psychophysical sensitivity to motion signals in extrastriate area MST of the macaque monkey. J Neurosci 14:4109-4124.

Chang T-Y, Thompson L, Doudlah R, Kim B, Sunkara A, Rosenberg A (2020) Optimized but not maximized cue integration for 3D visual perception. eNeuro 7:ENEURO.0411-0419.2019.

Cumming BG, Parker AJ (1997) Responses of primary visual cortical neurons to binocular disparity without depth perception. Nature 389:280-283.

Cumming BG, Parker AJ (1999) Binocular neurons in V1 of awake monkeys are selective for absolute, not relative, disparity. J Neurosci 19:5602-5618.

Cumming BG, Parker AJ (2000) Local disparity not perceived depth is signaled by binocular neurons in cortical area V1 of the Macaque. J Neurosci 20:4758-4767.

Cumming BG, Nienborg H (2016) Feedforward and feedback sources of choice probability in neural population responses. Curr Opin Neurobiol 37:126-132.

Dakin CJ, Rosenberg A (2018) Gravity estimation and verticality perception. Handb Clin Neurol 159:43-59.

DeAngelis GC, Uka T (2003) Coding of horizontal disparity and velocity by MT neurons in the alert macaque. J Neurophysiol 89:1094-1111.

Dodd JV, Krug K, Cumming BG, Parker AJ (2001) Perceptually bistable three-dimensional figures evoke high choice probabilities in cortical area MT. J Neurosci 21:4809-4821.

Elmore LC, Rosenberg A, DeAngelis GC, Angelaki DE (2019) Choice-related activity during visual slant discrimination in macaque CIP but not V3A. eNeuro 6:ENEURO.0248-0218.2019.

Fattori P, Breveglieri R, Raos V, Bosco A, Galletti C (2012) Vision for action in the macaque medial posterior parietal cortex. J Neurosci 32:3221-3234.

Fattori P, Raos V, Breveglieri R, Bosco A, Marzocchi N, Galletti C (2010) The dorsomedial pathway is not just for reaching: Grasping neurons in the medial parieto-occipital cortex of the macaque monkey. J Neurosci 30:342-349. 
832

833

834

835

836

837

838

839

840

841

842

843

844

845

846

847

848

849

850

851

852

853

854

855

856

857

858

859

860

861

862

863

864

865

866

867

868

869

870

871

872

873

874

875

876

877

878

879

880

881

Fisher NI (1995) Statistical analysis of circular data: Cambridge University Press.

Fisher NI, Lewis T, Embleton BJ (1993) Statistical analysis of spherical data: Cambridge University Press.

Freedman DJ, Assad JA (2006) Experience-dependent representation of visual categories in parietal cortex. Nature 443:85-88.

Gu Y, DeAngelis GC, Angelaki DE (2007) A functional link between area MSTd and heading perception based on vestibular signals. Nat Neurosci 10:1038-1047.

Gu Y, Angelaki DE, DeAngelis GC (2014) Contribution of correlated noise and selective decoding to choice probability measurements in extrastriate visual cortex. Elife 3.

Haefner RM, Gerwinn S, Macke JH, Bethge M (2013) Inferring decoding strategies from choice probabilities in the presence of correlated variability. Nat Neurosci 16:235-242.

Hanes DP, Schall JD (1996) Neural control of voluntary movement initiation. Science 274:427430.

Hartley R, Zisserman A (2003) Multiple view geometry in computer vision: Cambridge University Press.

Hastie T, Tibshirani R, Friedman J (2009) The elements of statistical learning: Data mining, inference, and prediction: Springer Science \& Business Media.

Holmes G (1918) Disturbances of visual orientation. The British Journal of Ophthalmology 2:449.

Holmes G, Horrax G (1919) Disturbances of spatial orientation and visual attention, with loss of stereoscopic vision. Archives of Neurology and Psychiatry 1:385-407.

Janssen P, Vogels R, Orban GA (2000) Three-dimensional shape coding in inferior temporal cortex. Neuron 27:385-397.

Kim B, Kenchappa SC, Sunkara A, Chang T-Y, Thompson L, Doudlah R, Rosenberg A (2019) Real-time experimental control using network-based parallel processing. eLife 8:e40231.

Lanzilotto M, Ferroni CG, Livi A, Gerbella M, Maranesi M, Borra E, Passarelli L, Gamberini M, Fogassi L, Bonini L, Orban GA (2019) Anterior intraparietal area: A hub in the observed manipulative action network. Cereb Cortex 29:1816-1833.

Law CT, Gold JI (2008) Neural correlates of perceptual learning in a sensory-motor, but not a sensory, cortical area. Nat Neurosci 11:505-513.

Lescroart MD, Gallant JL (2019) Human scene-selective areas represent 3D configurations of surfaces. Neuron 101:178-192 e177.

Liu S, Gu Y, DeAngelis GC, Angelaki DE (2013) Choice-related activity and correlated noise in subcortical vestibular neurons. Nat Neurosci 16:89-97.

Munoz DP, Wurtz RH (1995) Saccade-related activity in monkey superior colliculus. I. Characteristics of burst and buildup cells. J Neurophysiol 73:2313-2333.

Murata A, Gallese V, Luppino G, Kaseda M, Sakata H (2000) Selectivity for the shape, size, and orientation of objects for grasping in neurons of monkey parietal area AIP. J Neurophysiol 83:2580-2601.

Nakamura H, Kuroda T, Wakita M, Kusunoki M, Kato A, Mikami A, Sakata H, Itoh K (2001) From three-dimensional space vision to prehensile hand movements: The lateral intraparietal area links the area V3A and the anterior intraparietal area in macaques. J Neurosci 21:8174-8187.

Nakamura K, Colby CL (2000) Visual, saccade-related, and cognitive activation of single neurons in monkey extrastriate area V3A. J Neurophysiol 84:677-692.

Nguyenkim JD, DeAngelis GC (2003) Disparity-based coding of three-dimensional surface orientation by macaque middle temporal neurons. J Neurosci 23:7117-7128.

Nienborg H, Cumming BG (2006) Macaque V2 neurons, but not V1 neurons, show choice-related activity. J Neurosci 26:9567-9578.

Pani P, Theys T, Romero MC, Janssen P (2014) Grasping execution and grasping observation activity of single neurons in the macaque anterior intraparietal area. J Cogn Neurosci 26:23422355. 
Pause M, Freund HJ (1989) Role of the parietal cortex for sensorimotor transformation. Evidence from clinical observations. Brain Behav Evol 33:136-140.

Pause M, Kunesch E, Binkofski F, Freund HJ (1989) Sensorimotor disturbances in patients with lesions of the parietal cortex. Brain 112 ( Pt 6):1599-1625.

Premereur E, Van Dromme IC, Romero MC, Vanduffel W, Janssen P (2015) Effective connectivity of depth-structure-selective patches in the lateral bank of the macaque intraparietal sulcus. PLoS Biol 13:e1002072.

Rosenberg A, Angelaki DE (2014a) Gravity influences the visual representation of object tilt in parietal cortex. J Neurosci 34:14170-14180.

Rosenberg A, Angelaki DE (2014b) Reliability-dependent contributions of visual orientation cues in parietal cortex. Proc Natl Acad Sci U S A 111:18043-18048.

Rosenberg A, Cowan NJ, Angelaki DE (2013) The visual representation of 3D object orientation in parietal cortex. J Neurosci 33:19352-19361.

Rushworth MF, Nixon PD, Passingham RE (1997) Parietal cortex and movement. I. Movement selection and reaching. Exp Brain Res 117:292-310.

Sanada TM, DeAngelis GC (2014) Neural representation of motion-in-depth in area MT. J Neurosci 34:15508-15521.

Schwemmer MA, Fairhall AL, Deneve S, Shea-Brown ET (2015) Constructing precisely computing networks with biophysical spiking neurons. J Neurosci 35:10112-10134.

Seilheimer RL, Rosenberg A, Angelaki DE (2014) Models and processes of multisensory cue combination. Curr Opin Neurobiol 25:38-46.

Shushruth S, Mazurek M, Shadlen MN (2018) Comparison of decision-related signals in sensory and motor preparatory responses of neurons in area LIP. J Neurosci 38:6350-6365.

Taira M, Tsutsui KI, Jiang M, Yara K, Sakata H (2000) Parietal neurons represent surface orientation from the gradient of binocular disparity. J Neurophysiol 83:3140-3146.

Thomas OM, Cumming BG, Parker AJ (2002) A specialization for relative disparity in V2. Nat Neurosci 5:472-478.

Tsutsui K, Sakata H, Naganuma T, Taira M (2002) Neural correlates for perception of 3D surface orientation from texture gradient. Science 298:409-412.

Tsutsui K, Jiang M, Sakata H, Taira M (2003) Short-term memory and perceptual decision for three-dimensional visual features in the caudal intraparietal sulcus (area CIP). J Neurosci 23:5486-5495.

Tsutsui K, Jiang M, Yara K, Sakata H, Taira M (2001) Integration of perspective and disparity cues in surface-orientation-selective neurons of area CIP. J Neurophysiol 86:2856-2867.

Van Dromme IC, Premereur E, Verhoef B-E, Vanduffel W, Janssen P (2016) Posterior parietal cortex drives inferotemporal activations during three-dimensional object vision. PLOS Biol 14:e1002445.

Van Essen DC, Lewis JW, Drury HA, Hadjikhani N, Tootell RB, Bakircioglu M, Miller MI (2001) Mapping visual cortex in monkeys and humans using surface-based atlases. Vision Res 41:1359-1378.

Vaziri S, Carlson ET, Wang Z, Connor CE (2014) A channel for 3D environmental shape in anterior inferotemporal cortex. Neuron 84:55-62.

Zaidel A, DeAngelis GC, Angelaki DE (2017) Decoupled choice-driven and stimulus-related activity in parietal neurons may be misrepresented by choice probabilities. Nat Commun 8:715. 